\title{
Interfering with contextual fear memories by post-reactivation administration of propranolol in mice: a series of null findings
}

\author{
Wouter R. Cox ${ }^{1}$, Leonidas Faliagkas², Rolinka van der Loo $^{2}$, Sabine Spijker ${ }^{2}$, Merel Kindt', \\ Priyanka Rao-Ruiz ${ }^{2 *}$ \\ ${ }^{1}$ Department of Psychology, Clinical Psychology, University of Amsterdam, Amsterdam, The \\ Netherlands \\ ${ }^{2}$ Department of Molecular and Cellular Neurobiology, Center for Neurogenomics and Cognitive \\ Research, Amsterdam Neuroscience, Vrije Universiteit Amsterdam, Amsterdam, The Netherlands \\ * Correspondence: \\ Priyanka Rao-Ruiz \\ p.rao@vu.nl
}

Keywords: Reconsolidation, Memory, Contextual Fear, Propranolol, Hippocampus, Rodents

\begin{abstract}
Post-reactivation amnesia of contextual fear memories by blockade of noradrenergic signaling has been shown to have limited replicability in rodents. This is usually attributed to several boundary conditions that gate the destabilization of memory during its retrieval. However, how these boundary conditions can be overcome, and what neural mechanisms underlie post-reactivation changes in contextual fear memory remain largely unknown. Here, we report a series of experiments in a contextual fear conditioning paradigm in mice, that were aimed at elucidating these matters. Towards this overarching goal, we first attempted to obtain a training paradigm that would consistently result in a contextual fear memory that could be destabilized upon reactivation, enabling robust amnesia by administration of propranolol. Unexpectedly, our attempts were unsuccessful to this end.
\end{abstract}

Specifically, over a series of 11 experiments (including replicates) in which we varied different parameters of the fear acquisition procedure and administered propranolol or anisomycin, at best small and inconsistent effects were observed. These null findings are surprising, given that the training paradigms we implemented were previously shown to be vulnerable to post-reactivation amnestic agents. Additionally, we found that propranolol did not alter memory retrieval-induced 
neural activity, as measured by the number of c-Fos ${ }^{+}$cells in the hippocampal dentate gyrus. Together, our findings illustrate the elusive nature of reactivation-dependent changes of non-human fear memory and underscore the need for better control over genetic and environmental factors that may influence behavioral outcomes of commonly used mouse strains.

\section{Introduction}

Extensive neuroscientific evidence shows that noradrenergic signaling plays a pivotal role in emotional memory plasticity (Joëls et al., 2011). At the occurrence of an emotionally arousing event, noradrenergic transmission in the basolateral amygdala stimulates projections to downstream brain regions, such as the hippocampus, leading to the event being firmly stored in long-term memory (McGaugh, 2015, 2018; Roozendaal \& Hermans, 2017). Convincing demonstrations of this crucial involvement of the noradrenergic system in memory consolidation come from studies, showing that administering noradrenaline, and non-selective noradrenergic antagonists such as propranolol, shortly after fear learning leads to intensified and weakened memory expression, respectively (e.g., Gallagher et al., 1977; Liang et al., 1986; Ji et al., 2003; LaLumiere et al., 2003).

Interestingly, somewhat more recent empirical work has shown that noradrenaline is not only needed for the initial formation of emotional memory, but plays an equally important role in the persistence of memory after its reactivation, a process often referred to as reconsolidation. Building on earlier demonstrations of post-reactivation memory loss in the late nineteen-sixties (Misanin, Miller, \& Lewis, 1968), an influential study by Przybyslawski et al. (1999) showed that systemic injections of propranolol after not only encoding - but also reactivation - of emotional memories leads to impaired memory expression $48 \mathrm{~h}$ later. Further experimentation of this post-reactivation amnesia seemed to solidify the idea of a shared underlying mechanism between memory consolidation and "reconsolidation". Like the formation of new memories, protein synthesis was 
shown to be needed for the persistence of memory expression after reactivation (Nader, Schafe, \& Le Doux, 2000). Hence, it is argued that propranolol interferes with the creation of new plasticity-related proteins, specifically by preventing noradrenaline from activating the transcription factor cAMP response element-binding protein (CREB) (Thonberg, Fredriksson, Nedergaard, \& Cannon, 2002), such that fear memories are not (fully) restabilized in long-term memory (Przybyslawski et al., 1999).

However, the existence of reconsolidation is an issue of considerable debate (Elsey, van Ast, \& Kindt, 2018; Gisquet-Verrier \& Riccio, 2018). For example, despite the apparent similarities between memory consolidation and reconsolidation, there also seem to be differences, including the involvement of the noradrenergic system. Both processes do not seem to involve identical brain regions and circuits (Alberini, 2005), and several studies suggest that propranolol may only impact reactivated memories, and not necessarily the formation of new memories (Dębiec \& Ledoux, 2004; Villain et al., 2016). Furthermore, the exact mechanisms by which propranolol interferes with fear memories at the level of memory traces (i.e., the neuronal ensemble that encodes and stores memories) remains largely unknown. This is especially true for hippocampus-dependent contextual fear memories, for which the involvement of the noradrenergic system is not as well defined as it is for amygdala-dependent cued fear memories (McGaugh, 2002; Roozendaal, McEwen, \& Chattarji, 2009). It is evident that synaptic function in the hippocampus can be bidirectionally modulated by the application of adrenergic agonists and antagonists (Gelinas \& Nguyen, 2005; Giustino \& Maren, 2018). A recent study demonstrated that propranolol has an acute effect on the expression of contextual fear memories and alters memory traces in the dorsal dentate gyrus (DG), basolateral amygdala and pre-frontal cortex when administered before memory reactivation (Leal Santos et al., 2021). However, how propranolol may induce amnesia and affect memory traces after drug washout cannot be inferred as no lasting fear-reducing effects of propranolol were found in this study. For 
these reasons, gaining insights in the exact neural underpinnings of propranolol's effects on reactivated memories, especially contextual fear memories, remains of significant interest.

Next to fundamental insights into the involvement of the noradrenergic system in memory plasticity, another highly relevant attribute of research showing reductions in fear responding by post-reactivation administration of propranolol is clinical application. A procedure that can be employed to interfere with fear memories would have great utility in the treatment of anxiety disorders and posttraumatic stress disorder. Extinction-based treatments, that currently dominate clinical practice, lead to the creation of a new memory trace that competes with the fear memory, instead of altering it directly, resulting in high relapse rates through a variety of mechanisms (Bouton, 2002). Administering amnestic agents, such as propranolol, in close temporal proximity of memory reactivation seems to result in more durable reductions in fear responding (Bustos, Maldonado, \& Molina, 2006; Dębiec \& Ledoux, 2004; Dębiec, LeDoux, \& Nader, 2002; Duvarci \& Nader, 2004, but see Eisenberg \& Dudai, 2004; Lattal \& Abel, 2004). Crucially, post-reactivation administration of propranolol has also shown to interfere with fear memories in a human fear conditioning paradigm (Kindt et al., 2009; Soeter and Kindt, 2010, 2011, 2012a, 2012b, 2015b; Sevenster et al., 2012, 2013, 2014; Kindt \& Soeter, 2018, but see Bos et al., 2014; Schroyens et al., 2017; Chalkia et al., 2019), bringing the development of a new, effective treatment for disorders of emotional memory an important step closer.

Translation of these findings to real-life anxiety disorders and posttraumatic stress disorder remains a great challenge (Kindt, 2014). At present, both promising (Brunet et al., 2008, 2011, 2018; Kindt \& van Emmerik, 2016; Soeter \& Kindt, 2015a) as well as disappointing (Elsey et al., 2020; Roullet et al., 2021; Wood et al., 2015) results have been reported to treat real-life fears that have been acquired outside the laboratory. These relatively mixed observations have often been attributed to the subtle conditions under which amnestic agents interfere with fear memories (Elsey et al., 2020, 2018). Reactivation of a memory may not be sufficient, but only if a violation of a fear-related 
expectancy occurs (e.g., not receiving an anticipated aversive stimulus) does administration of amnestic agents lead to reduced fear responding (Lee, 2009; Pedreira, Pérez-Cuesta, \& Maldonado, 2004; Sevenster et al., 2012). However, with prolonged exposure to the feared stimuli, fear memories could enter a "limbo" state or a new extinction memory is created, such that protein synthesis inhibitors do not target the original fear memory anymore (Merlo, Milton, Goozee, Theobald, \& Everitt, 2014; Sevenster et al., 2014). Complicating matters even further is that the duration of reactivation at which amnestic agents can reduce the later expression of fear seems to be intricately dependent on memory strength and memory age, such that stronger and older fear memories might require longer exposure to the feared stimulus (Bustos, Maldonado, \& Molina, 2009; Suzuki et al., 2004). To date, there is no measure available to adjust the memory reactivation sessions based on the aforementioned factors that vary greatly from patient to patient. A procedure through which the challenges posed by the boundary conditions could be circumvented would therefore be of great value.

Thus, in a quest to (i) elucidate the effects of propranolol on neuronal ensembles activated by the retrieval of contextual fear memory and (ii) discover how boundary conditions of postreactivation amnesia can be overcome, we performed a series of experiments in an animal fear conditioning paradigm. Towards these goals, our first aim was to obtain a behavioral paradigm that would consistently show reactivation-induced amnesia by the administration of propranolol after contextual memory reactivation. We performed a series of 11 experiments (including replicates) with varied training protocols, and post-reactivation amnestic agents (propranolol and anisomycin). However, these attempts to induce robust post-reactivation amnesia were unsuccessful, such that no further experimentation was possible. Our behavioral data sharply contradict earlier successful reports (Abrari, Rashidy-Pour, Semnanian, \& Fathollahi, 2008; Liu et al., 2015; Muravieva \& Alberini, 2010), but is reminiscent of a recent series of likewise unsuccessful experiments in rats that includes the administration of propranolol, midazolam, and cycloheximide (Schroyens, Alfei, 
Schnell, Luyten, \& Beckers, 2019). We build on these data, now using a different species (i.e., mice), to demonstrate that post-reactivation amnesia of contextual fear memories by systemic administration of propranolol does not work with flawless fidelity. In addition, no effect of the commonly used amnestic agent anisomycin (Barbacid \& Vazquez, 1974) was found, indicating that we were unable to trigger memory destabilization and subsequent restabilization using training protocols that were previously successful in this respect. At the molecular level, propranolol had no effect on retrievalinduced activity of putative memory trace cells in the hippocampal Dentate Gyrus (DG). Together, the data provided here comprises a valuable resource for future research aimed at elucidating the precise conditions under which contextual fear memories can be destabilized and manipulated upon their reactivation. Furthermore, it shows the need for systematic control over genetic and environmental factors that may indirectly influence behavioral and cellular outcomes of experimental animals over time.

\section{Materials and Methods}

\subsection{General procedures.}

2.1.1. Animals. Male C57BI6/J inbred mice (8-10 weeks of age), from Charles River France, were individually housed with enrichment and kept on a $12 \mathrm{~h}$ light/dark cycle with food and water available ad libitum. The mice habituated to their home cage for two weeks prior to experimentation. All procedures were carried out during the light phase, between 9 A.M. and 12 P.M., unless otherwise specified. Prior to the start of the experiment, the mice underwent three consecutive days of handling (2-3 minutes per mouse) to habituate the animals to being held and restrained by the experimenter. All experiments were performed in accordance with Dutch law and licensing agreements using protocols ethically approved by the Animal Ethical Committee of the Vrije 
Universiteit Amsterdam.

2.1.2. Apparatus. The experiments were executed in two automated fear conditioning systems: (i) TSE Fear Conditioning System for Small Rodents (TSE systems, Germany) in Experiments 1-2 (e.g., Rao-Ruiz et al., 2011; Vegh et al., 2014) and (ii) Med Associates Video Fear Conditioning System (Sandown Scientific, UK) in Experiments 3-8 (e.g., Anagnostaras et al., 2010; GoutyColomer et al., 2016). The mice underwent contextual fear conditioning, reactivation, and retrieval in a conditioning chamber with a stainless-steel grid floor, constant house light (TSE: 100-500 lx, Med Associates: White light: 450-650 nm and near-infrared light: $940 \mathrm{~nm}$ ) and background white noise (TSE: $68 \mathrm{~dB}$, Med Associates: $50 \mathrm{~dB}, 5000 \mathrm{~Hz}$ ). The fear conditioning box was placed in a sound attenuating outer chamber. The apparatus was cleaned with $70 \%$ ethanol prior to each session.

2.1.3. Drugs. Propranolol $\mathrm{HCl}$ (Sigma-Aldrich) was dissolved in saline at a ratio of $2 \mathrm{mg} / 1 \mathrm{~mL}$, unless otherwise specified, and anisomycin (Sigma-Aldrich) at a ratio of $30 \mathrm{mg} / 1500 \mathrm{~mL}$. The mice in the experimental groups received $10 \mathrm{mg}$ per $\mathrm{kg}$ body weight of propranolol (Przybyslawski et al., 1999; Villain et al., 2016) or anisomycin at $150 \mathrm{mg}$ per kg body weight (Rao-Ruiz et al., 2011; Suzuki et al., 2004). The mice in the control group received the same volume of saline. The drugs were administered immediately after memory reactivation through intraperitoneal injections in all experiments.

2.1.4. Data analysis. Freezing, defined as the cessation of all movement except respiration and heartbeat, was used as a measure of fear memory and scored using automated algorithms in the TSE (Rao-Ruiz et al., 2011) or Med Associates systems (Anagnostaras et al., 2010; Gouty-Colomer et al., 2016). For the TSE chamber, a time threshold of $1 \mathrm{~s}$ was used for automatically recorded "freezing" 
behavior (i.e., no detected change in sensor status for $1 \mathrm{~s}$ in $\mathrm{X}, \mathrm{Y}$ and $\mathrm{Z}$ axes). For the Med associates chamber, freezing was measured by Video Freeze Software (Anagnostaras et al., 2010) using a linear method with a motion threshold of 18 a.u., sample rate of 30 frames per second and a minimum freeze duration of 30 frames. Freezing data is presented as a percentage of the total reactivation/retrieval test time.

The statistical analyses were carried out using the Statistical Package for the Social Sciences (SPSS) version 25.0 (Armonk, NY: IBM Corp.). To assess whether the administration of amnestic drugs (propranolol or anisomycin) after memory reactivation results in an attenuation of fear responding, we performed a mixed ANOVA with Drug (amnestic drug vs. saline) as betweensubjects factor and Session (reactivation, retrieval) as within-subjects factor. When relevant, we performed additional independent t-tests. To make sure that the null findings cannot be attributed to any animals not having acquired contextual fear, we performed additional analyses in which only animals exhibiting a freezing level of $10 \%$ or higher during reactivation were included (see Figure S1). This inclusion of mice based on freezing levels did not lead to different results, unless reported otherwise in the main manuscript.

\subsection{Specific procedures.}

\subsubsection{Experiment 1.}

2.2.1.1. Contextual fear conditioning. Mice were placed in the fear conditioning chamber, and after $180 \mathrm{~s}$, received a $2 \mathrm{~s} 0.7 \mathrm{~mA}$ foot shock as the unconditioned stimulus (US). Thirty seconds later, the mice were removed from the box and placed in their home cage.

2.2.1.2. Memory reactivation. Twenty-four hours afterwards, to reactivate the fear memory, the mice were placed in the conditioning box for $180 \mathrm{~s}$ without delivery of the US. Immediately 
thereafter, the mice were weighed and received either saline or propranolol in a quasi-random fashion (i.e., alternation) and then returned to their home cage.

2.2.1.3. Memory retrieval. Forty-eight hours after memory reactivation (Dębiec \& Ledoux, 2004), mice were placed in the conditioning box for $180 \mathrm{~s}$ in the absence of the unconditioned stimulus and returned to their home cage immediately afterwards. One mouse was excluded due to a procedural error (Experiment 1b, saline condition).

2.2.1.4. Immunohistochemistry. Ninety minutes after memory retrieval, 5 animals from each experimental group (saline or propranolol), were randomly selected and transcardially perfused with ice-cold PBS, followed by 4\% paraformaldehyde (Sigma-Aldrich Chemie N.V., The Netherlands). Forty micrometer coronal sections of the hippocampus were collected serially using a freezing microtome (Leica, Wetzlar, Germany; SM 2000R) and stored in PBS with $0.02 \% \mathrm{NaN}_{3}$ at $4{ }^{\circ} \mathrm{C}$ until further use. Approximately 8-10 free floating sections across the rostrocaudal axis of the dorsal DG were used for immunohistochemical stainings (described in Rao-Ruiz et al., 2019) using a primary antibody against the Immediate Early Gene c-Fos (1:500, sc-52, Santa Cruz, Germany) and a corresponding Alexa-conjugated secondary antibody (1:400, anti-rabbit Alexa 488, Life Technologies, The Netherlands). Nuclear staining was performed using DAPI (300 nmol/L, Thermo Fisher Scientific, The Netherlands). Sections were mounted on slides and coverslipped using Polyvinyl alcohol mounting medium with DABCO®, antifading (Merck KGaA, Germany).

2.2.1.5. Confocal microscopy, cell counting and data analysis. A Nikon Instruments A1 Confocal Laser Microscope with NIS-Elements C Software was used to make approximately 8-10 z-stacks of the DG/animal at 10x magnification by an experimenter blinded to the experimental condition. Images were imported to Fiji (Version 1.0) where they were digitally merged to form composite 
images. Individual c-Fos ${ }^{+}$cells in the DG were manually marked, counted using the Cell Counter plugin in Fiji, and averaged/1.3 mm section for each animal. Representative images were edited in Image J to generate 2D projections of z-stacks, and all images were treated identically. The effect of drug administration on average number of ${\mathrm{c}-\mathrm{Fos}^{+}}^{+}$cells was analyzed using unpaired t-tests in Prism 8 (version 8.4.3).

\subsubsection{Experiment 2.}

2.2.2.1. Contextual fear conditioning. During fear conditioning, a $1 \mathrm{~mA}$ foot shock was administered (instead of a $0.7 \mathrm{~mA}$ foot shock as described in Experiment 1). All other procedures were the same as in Experiment 1.

2.2.2. Immunohistochemistry. Seven animals randomly selected from each experimental group (saline or propranolol) were used for c-Fos immunohistochemistry as described in Experiment 1.

2.2.3. Experiment 3. In addition to administering propranolol in the same way as in Experiment 12, we also included a condition in which this drug was dissolved at a ratio of $1 \mathrm{mg} / 1 \mathrm{~mL}$ (Villain et al., 2016), and injected with double the volume. All other procedures were the same as in Experiment 2.

2.2.4. Experiment 4. The mice were kept on a reversed, $12 \mathrm{~h}$ light/dark scale, such that all experimental procedures (i.e., habituation, handling, conditioning, reactivation, retrieval) took place during the dark phase, between 9 A.M. and 12 P.M. All other behavioral procedures were the same as in Experiment 2. 


\subsubsection{Experiment 5.}

2.2.5.1. Background contextual fear conditioning. The mice were placed in the conditioning chamber, and after $120 \mathrm{~s}$, a tone $(5 \mathrm{kHz}, 75 \mathrm{~dB})$ was presented for $30 \mathrm{~s}$, followed by a $2 \mathrm{~s} 1 \mathrm{~mA}$ foot shock. Thirty seconds after shock discontinuation, the mice were removed from the conditioning chamber and returned to their home cage.

2.2.5.2. Memory reactivation. Forty-eight hours after conditioning, mice were placed back in the conditioning box for $90 \mathrm{~s}$ without tone or shock delivery.

2.2.5.3. Memory retrieval. Forty-eight hours after reactivation, mice were placed in the conditioning box for $180 \mathrm{~s}$ without delivery of a tone or shock, as a test of contextual fear.

2.2.6. Experiment 6. In addition to propranolol, we included a condition in which animals received anisomycin. All other behavioral procedures were the same as in Experiment 2.

\subsubsection{Experiment 7 .}

2.2.7.1. Contextual fear conditioning. The procedure was based on an earlier study that has shown post-reactivation memory loss in rats (Schmidt et al., 2017). During conditioning, the mice were placed in the conditioning chamber and, after $120 \mathrm{~s}$, received a total of three $0.5 \mathrm{~mA}$ shocks with a 2 $\mathrm{s}$ duration. The shocks were administered with regular intervals of $30 \mathrm{~s}$ in between each shock. Thirty seconds after discontinuation of the last US, the mice were removed from the conditioning chamber, and returned to their home cage. All other procedures were the same as in Experiment 6.

\subsubsection{Experiment 8.}


2.2.8.1. Contextual fear conditioning. Instead of three $0.5 \mathrm{~mA}$ shocks, the mice received three 0.3 mA shocks. All other procedures were the same as in Experiment 7.

\section{Results}

3.1. Experiment 1. In Experiment 1, we tested whether the administration of propranolol after reactivation of a contextual fear memory eliminates expression of fear during a retrieval test $48 \mathrm{~h}$ later, using a behavioral protocol that has been shown to result in post-reactivation amnesia by anisomycin (Rao-Ruiz et al., 2011; Suzuki et al., 2004). We observed that propranolol did not lead to reduced fear responding (Drug $\times$ Session, $F_{1,18}=0.021, p=0.887$, Figure $\left.1 \mathrm{~B}\right)$. This finding is not in line with post-reactivation amnesia using propranolol in previous studies (e.g., Taherian et al., 2014). Therefore, to ascertain that the present finding was not a false negative, we performed an exact repetition of the experiment (Experiment 1b). 
A
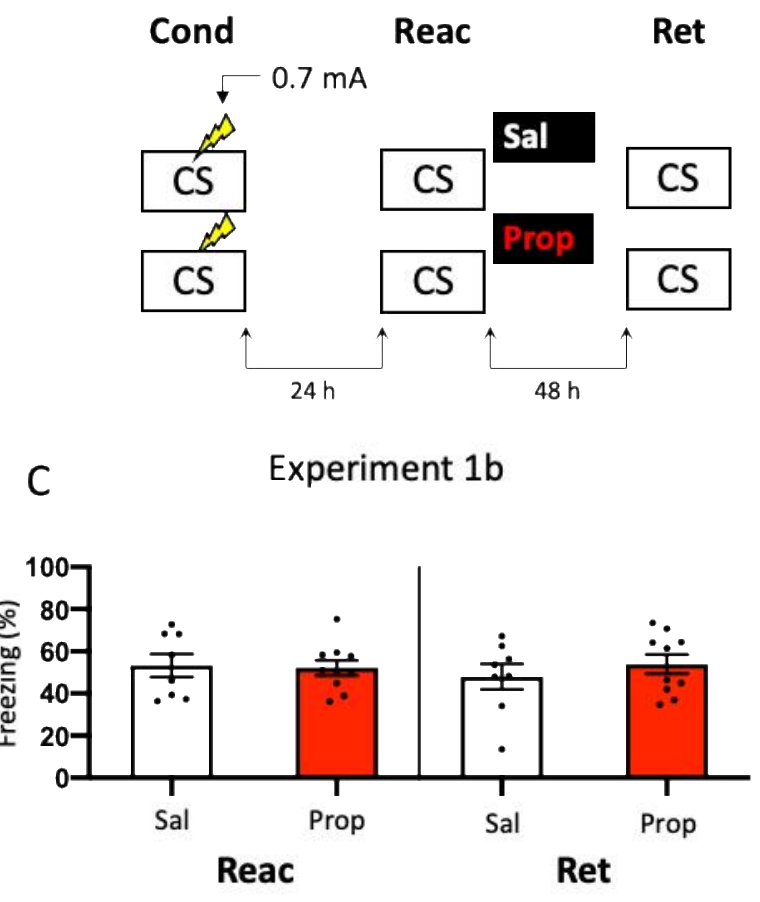

E

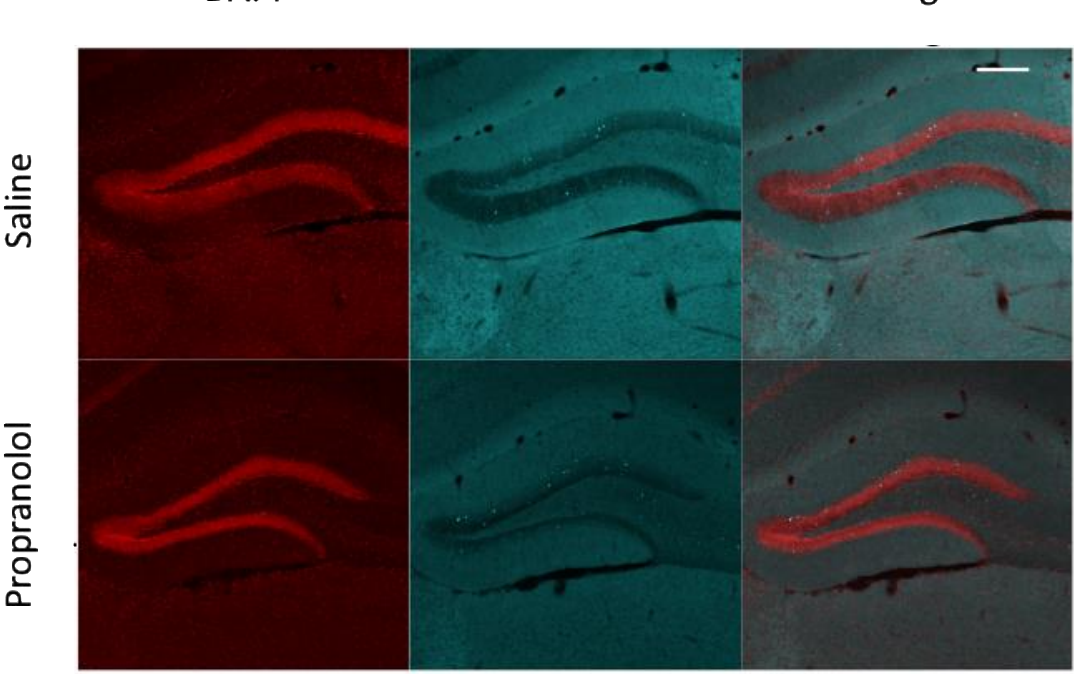

B

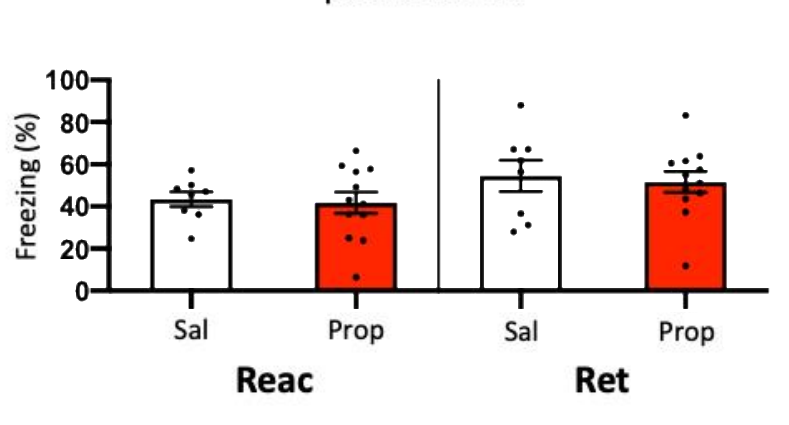

D

Experiment 1 (collapsed)

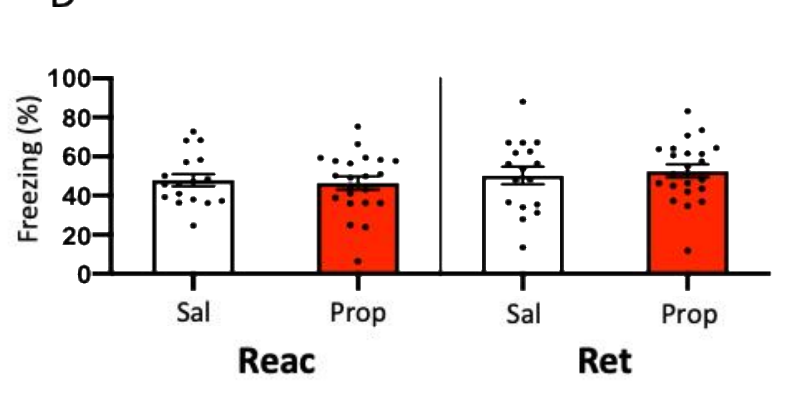

F

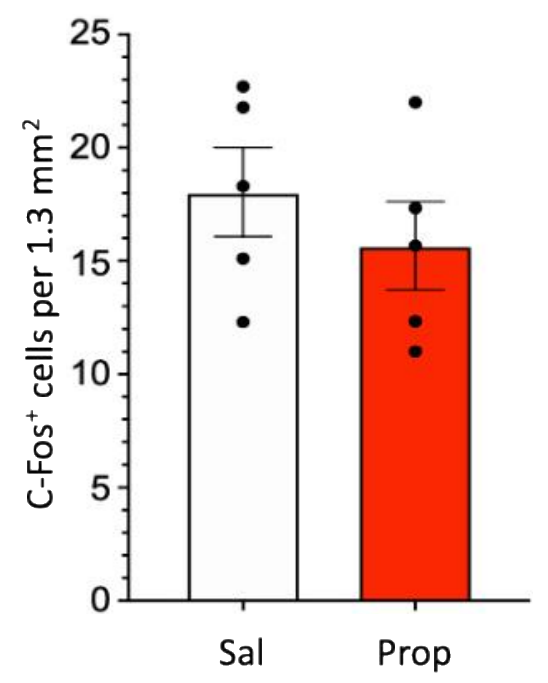

Fig. 1. No effect of propranolol on reactivated memories that were acquired with a single $0.7 \mathrm{~mA}$ shock during contextual fear conditioning. (A) Design of Experiment 1. Mice received one $0.7 \mathrm{~mA}$ shock during fear conditioning. One day later, saline $(n=8)$ or propranolol $(n=12)$ was administered after reactivation. Forty-eight hours afterwards, retrieval of contextual fear was assessed. Cond $=$ conditioning, Reac $=$ reactivation, Ret $=$ retrieval, $\mathrm{CS}=$ conditioned stimulus $($ context $)$, Sal $=$ saline, Prop $=$ propranolol. $(\mathbf{B})$ Results of Experiment 1a. Average percentage of freezing during memory reactivation is displayed on the left, and freezing during contextual memory retrieval on the right pane of the column chart (saline in white bars, propranolol in red bars). Error bars represent SEM. Filled circles indicate individual animals. (C) Results of Experiment 1b (saline, $\mathrm{n}=9$; propranolol, $\mathrm{n}=10$ ). Experiment 1b was a direct replication of Experiment 1a. (D) Collapsed results of Experiment 1a and Experiment 1b. (E) Representative images of the DG 90 minutes after memory retrieval from fear conditioned mice that received either saline $(n=5)$, or propranolol $(n=5)$ post-reactivation

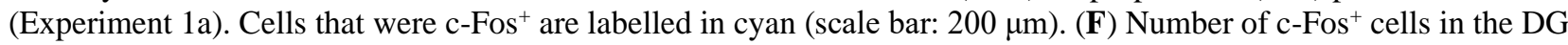
per $1.3 \mathrm{~mm}^{2}$. Error bars represent SEM. Filled circles indicate individual animals. 
Similar to Experiment 1a, in Experiment 1b there was no effect of propranolol on the pattern of freezing levels from reactivation to the retrieval test two days later (Drug $\times$ Session, $F_{1,16}=0.582$, $p=0.457$, Figure 1C). To ensure that the two present null findings are not a reflection of insufficient power, we collapsed the data of Experiment 1a and 1b, and repeated the analysis. No effect was observed on the collapsed data either $\left(F_{1,36}=0.277, p=0.602\right.$, Figure 1D).

Although no amnestic effect of propranolol was observed at the level of memory expression, we sought to determine if the manipulation of noradrenergic signaling after memory reactivation affected retrieval-induced neural activity in the DG, where memory traces for contextual fear have been previously identified (Denny et al., 2014; Liu et al., 2012; Rao-Ruiz et al., 2019). To this end, we measured the number of c-Fos ${ }^{+}$cells, a molecular correlate of neural activity, 90 minutes after context memory retrieval in the granule cell layer of the DG. In line with our behavioral results, no significant differences were observed in the number of activated DG neurons $\left(\mathrm{c}-\mathrm{Fos}^{+}\right)$between experimental groups ( $t_{8}=0.856, p=0.416$, Figures $1 \mathrm{E}$ and $\mathrm{F}$ ). Together, these data indicate that post-reactivation administration of propranolol does not affect subsequent retrieval or the activation of hippocampal neurons that are considered to be the cellular substrate of memory using the current experimental protocol.

3.2. Experiment 2. Since previous research has shown (though not consistently, Taherian et al., 2014) that memory strength modulates post-reactivation amnesia (Suzuki et al., 2004), we hypothesized that fear acquisition in Experiment 1 may not have been robust enough with the current protocol. Indeed, close inspection of the data of Experiment 1 shows that the freezing levels were considerably lower than in a previous study which involved the same mouse strain, laboratory space, behavioral system and training and reactivation protocols (Rao-Ruiz et al., 2011, see Fig. S2 for the results of Experiment 1 with freezing measured using a time threshold of $5 \mathrm{~s}$ as in this previous 
study). Therefore, to induce stronger fear memories we administered a single, higher intensity, i.e. 1 $\mathrm{mA}$, foot shock during conditioning.

However, no reduction in fear responding by propranolol was found in this experiment either (Drug $\times$ Session, $F_{1,14}=1.200, p=0.292$, Figure $2 \mathrm{~B}$ ). To make sure that the results were not a false negative, we repeated the experiment (Experiment 2b). Again, no significant effect was observed $\left(\right.$ Drug $\times$ Session, $F_{1,16}=2.523, p=0.132$, Figure $2 \mathrm{C}$ ). When the data of Experiment $2 \mathrm{a}$ and $2 \mathrm{~b}$ were collapsed we did find a trend for reduction in freezing from reactivation to retrieval in the propranolol relative to the saline condition (Drug $\times$ Session, $F_{1,32}=3.827, p=0.059$, Figure 2D). Notably, raising the foot shock level in Experiment 2 did not lead to higher freezing levels during reactivation than in Experiment $1(t 70=0.876, p=0.384$, compare Figures $1 \mathrm{D}$ and $2 \mathrm{D})$. Furthermore, this reduction in fear responding by post-reactivation administration of propranolol that appeared when the data of Experiment $2 \mathrm{a}$ and $2 \mathrm{~b}$ were collapsed is considerably smaller than in previous studies (e.g., Liu et al., 2015; Villain et al., 2016).

Nevertheless, since a marginal amnestic effect of propranolol on memory retrieval was observed, we further tested if neural activity in the DG was affected as well. However, as in Experiment 1, administration of propranolol had no effect on the number of DG neurons activated (cFos $\left.^{+}\right)$by memory retrieval $\left(t_{12}=1.046, p=0.316\right.$, Figure $2 \mathrm{E}$ and $\left.\mathrm{F}\right)$. We reasoned that the lack of an effect of post-reactivation propranolol on memory retrieval could be due to two factors: (i) limited efficacy of propranolol, or (ii) relatively unsuccessful triggering of memory destabilization. These two explanations were addressed in the subsequent experiments (3-5, and 6, respectively). 
A
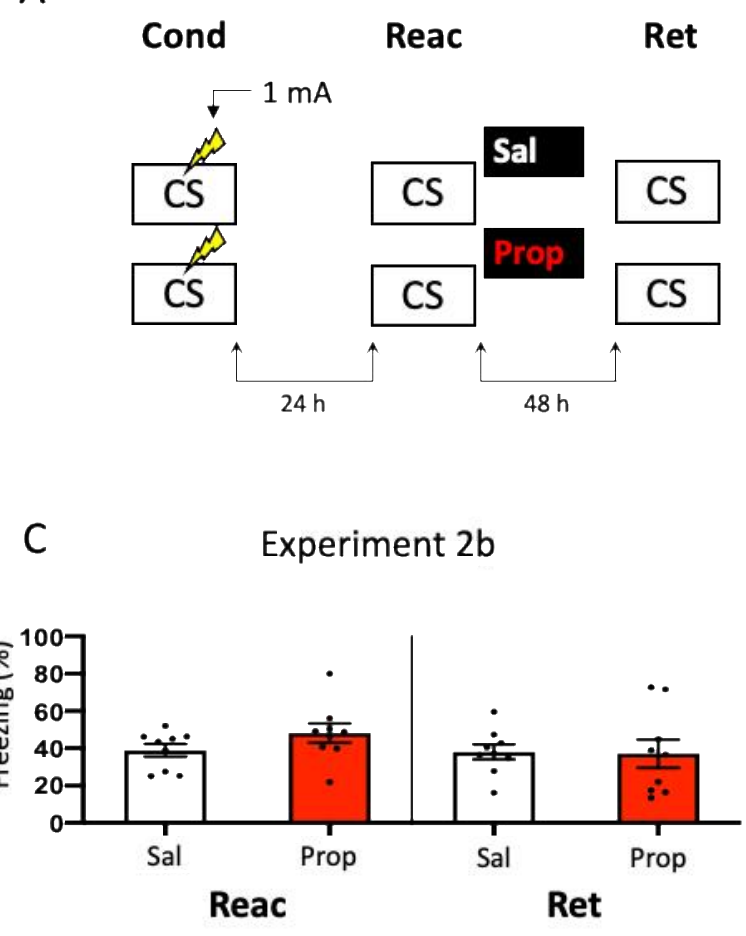

$\mathrm{E}$

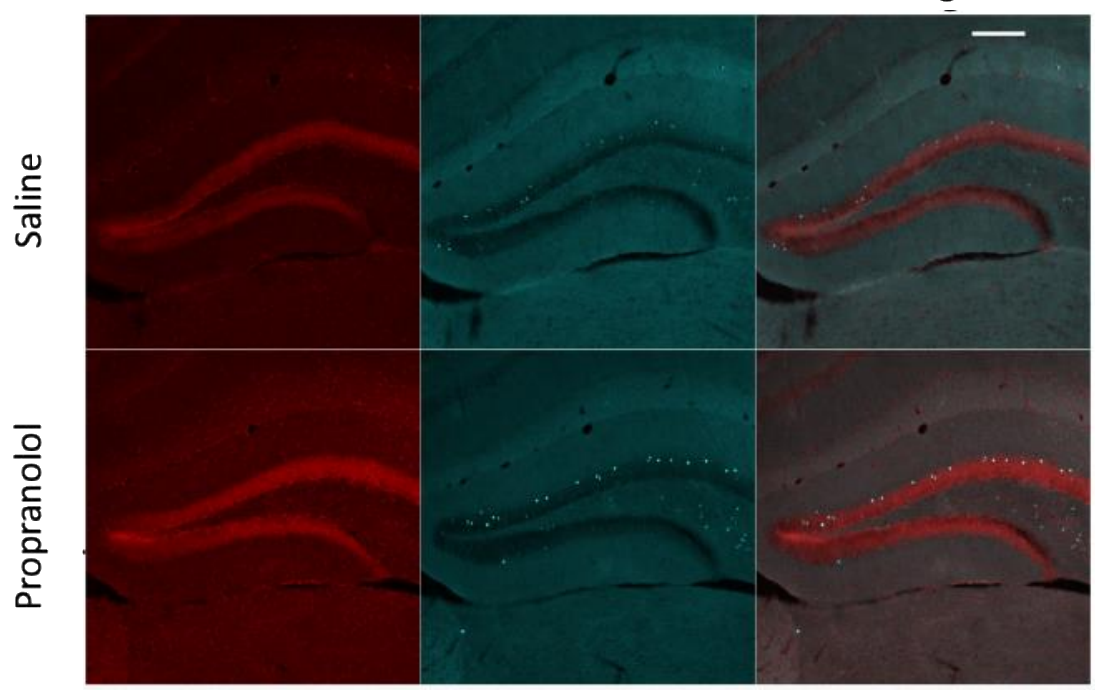

B

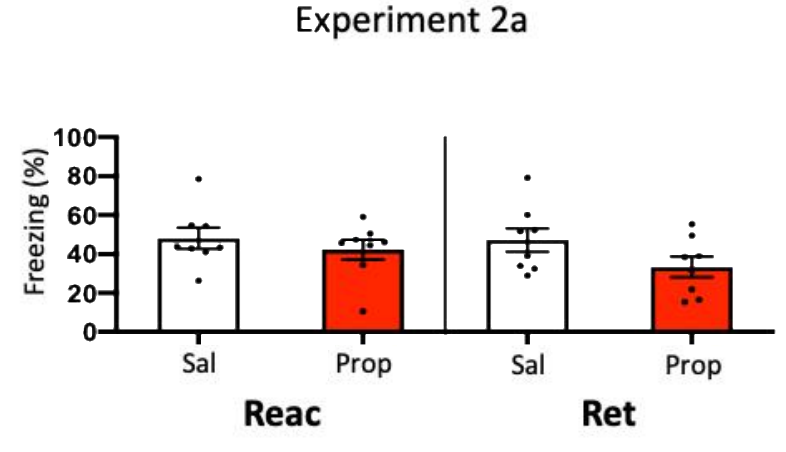

D Experiment 2 (collapsed)

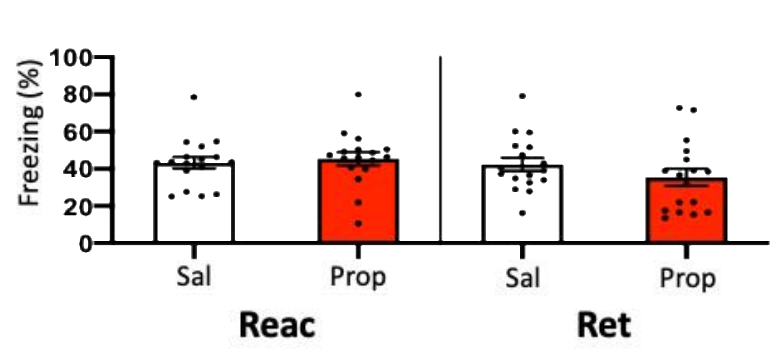

$\mathrm{F}$

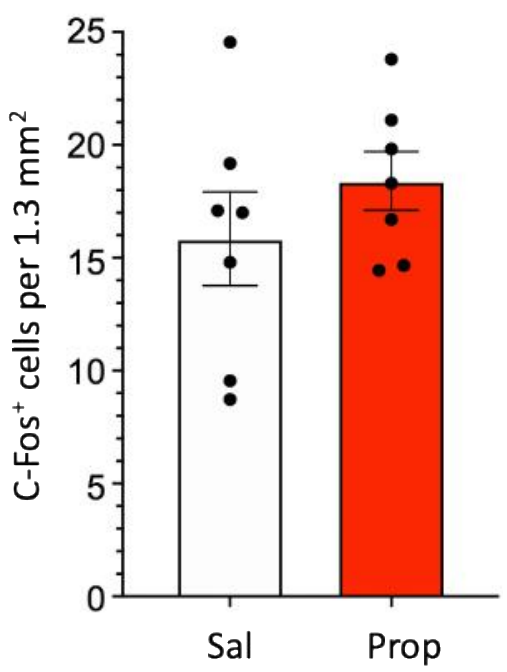

Fig. 2. A minor effect of propranolol on reactivated memories that were acquired with a single $1 \mathrm{~mA}$ shock during contextual fear conditioning. (A) Design of Experiment 2a. Mice received one $1 \mathrm{~mA}$ shock during fear conditioning. One day later, saline $(n=8)$ or propranolol $(n=8)$ was administered after reactivation. Forty-eight hours afterwards, retrieval of contextual fear was assessed. Cond $=$ conditioning, Reac $=$ reactivation, $\operatorname{Ret}=$ retrieval, $C S=$ conditioned stimulus (context), Sal $=$ saline, Prop $=$ propranolol. $($ B $)$ Results of Experiment 2a. Average percentage of freezing during memory reactivation is displayed on the left, and freezing during contextual memory retrieval on the right pane of the column chart. (saline in white bars, propranolol in red bars). Error bars represent SEM. Filled circles indicate individual animals. (C) Results of Experiment 2b (saline, $n=9$; propranolol, $n=9$ ). Experiment $2 \mathrm{~b}$ was a direct replication of Experiment $2 \mathrm{a}$. (D) Collapsed results of Experiment 2a and Experiment 2b. (E) Representative images of the DG 90 minutes after memory retrieval from fear conditioned mice that received either saline $(n=7)$, or propranolol $(n=7)$ post-reactivation (Experiment 2a). Cells that were c-Fos ${ }^{+}$are labelled in cyan (scale bar: $200 \mu \mathrm{m}$ ). (F) Number of c-Fos ${ }^{+}$cells in the DG per $1.3 \mathrm{~mm}^{2}$. Error bars represent SEM. Filled circles indicate individual animals. 
3.3. Experiment 3. As propranolol has lipophilic properties (Cruickshank, 1980), it is possible that a substantial amount of the drug binds to body fat upon injecton, such that noradrenergic signaling in the brain was only partially blocked in Experiment 2, resulting in reduced efficacy and relatively mild amnesia. Therefore, we performed an experiment that was the same as Experiment 2, but now also included a condition in which propranolol was dissolved in saline at half the ratio and injected in double the volume/body weight (i.e., to reduce binding to body fat without changing the dose). However, injecting propranolol in a larger volume of saline did not lead to a more robust decrease in fear responding (Drug $\times$ Session, $F_{2,24}=0.499, p=0.613$, Figure 3B).

A

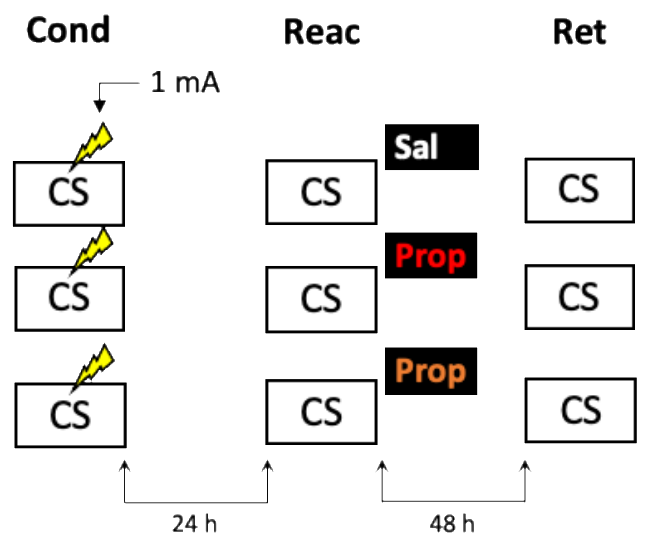

B

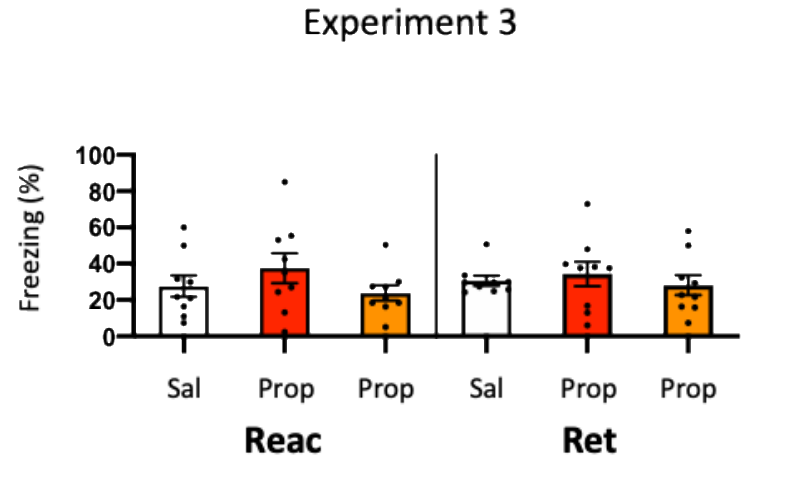

Fig. 3. No effect of propranolol - injected in two different volumes - on reactivated memories that were acquired with a single $1 \mathrm{~mA}$ shock during contextual fear conditioning. (A) Design of Experiment 3. Mice received one $1 \mathrm{~mA}$ shock during fear conditioning. One day later, mice received saline $(n=9)$, propranolol $(n=9)$, or propranolol dissolved in saline at half the ratio and injected in double the volume/body weight $(n=9)$ after reactivation. Forty-eight hours afterwards, retrieval of contextual fear was assessed. Cond $=$ conditioning, Reac $=$ reactivation, Ret $=$ retrieval, $\mathrm{CS}=$ conditioned stimulus (context), Sal $=$ saline, Prop $=$ propranolol. $($ B $)$ Results of Experiment 3. Average percentage of freezing during memory reactivation is displayed on the left, and freezing during contextual memory retrieval on the right pane of the column chart (saline in white bars, propranolol in red bars, and propranolol double volume in orange bars). Error bars represent SEM. Filled circles indicate individual animals.

3.4. Experiment 4. Another potential explanation for a small effect of propranolol in Experiment 2 might be that the experiments were performed during the light phase (i.e., the sleep cycle of mice). Previous research has shown that noradrenergic signaling from the locus coeruleus goes into timeouts during specific phases of the sleep cycle (Aston-Jones \& Bloom, 1981), such that synaptic 
plasticity is impaired (Cirelli \& Tononi, 2000). Therefore, in Experiment 2 the memories might have been reactivated and restabilized with little involvement of those receptors that propranolol specifically targets (i.e., $\beta$-adrenergic receptors, Johansen, Cain, Ostroff, \& Ledoux, 2011). Hypothetically, a more robust effect of propranolol on reactivated fear might be observed when memories are acquired and reactivating in the animals' wake-phase. Since rodents are nocturnal, we repeated Experiment 2, but now performed all experimental procedures in the animals' dark phase. Again, no fear reduction by propranolol was found (Drug $\times$ Session, $F_{1,22}=0.031, p=0.861$, Figure 4B). The freezing levels during reactivation were notably low compared to the previously performed experiments (e.g., Experiment 2), even after exclusion of mice with freezing levels below 10\% (Figure $\mathrm{S} 1 \mathrm{H}$ ). This may be attributable to light enhancing freezing levels (Warthen, Wiltgen, \& Provencio, 2011), so that when experiments are performed in the dark phase the animals freeze to a particularly low degree.

A

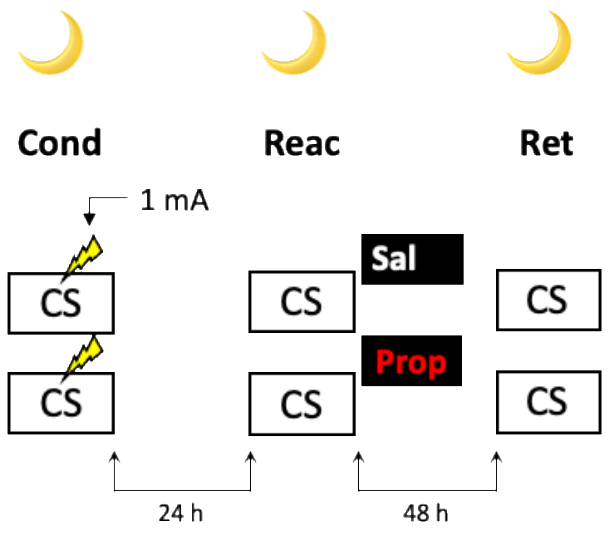

B

\section{Experiment 4}

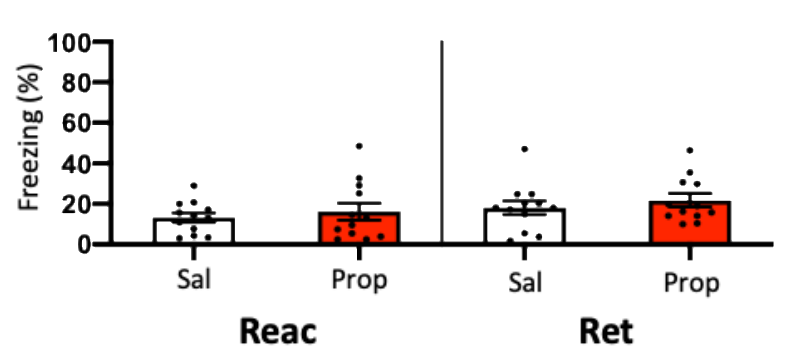

Fig. 4. No effect of propranolol on reactivated memories that were acquired with a single $1 \mathrm{~mA}$ shock during contextual fear conditioning in the animals' dark phase. (A) Design of Experiment 4. Mice received one $1 \mathrm{~mA}$ shock during fear conditioning. One day later, mice received saline $(n=12)$, propranolol $(n=12)$ after reactivation. Forty-eight hours afterwards, retrieval of contextual fear was assessed. All procedures took place in the animals' dark phase. Cond $=$ conditioning, Reac $=$ reactivation, $\mathrm{Ret}=$ retrieval, $\mathrm{CS}=$ conditioned stimulus $($ context $)$, Sal $=$ saline, Prop $=$ propranolol. (B) Results of Experiment 4. Average percentage of freezing during memory reactivation is displayed on the left, and freezing during contextual memory retrieval on the right pane of the column chart (saline in white bars, propranolol in red bars). Error bars represent SEM. Filled circles indicate individual animals. 


\subsection{Experiment 5. A last potential way to increase the efficacy of post-reactivation}

administration propranolol and to induce more robust amnesia could be to alter the way in which the contextual fear memory is processed. For example, previous research has shown differences in how foreground contextual fear conditioning and background contextual fear conditioning are encoded and the role of the hippocampus in each (Trifilieff et al., 2006). Furthermore, a study has shown postreactivation amnesia with propranolol in a background contextual fear conditioning paradigm (Muravieva \& Alberini, 2010). In the next experiment, we therefore aimed to induce amnesia by administering propranolol after reactivation of a memory that was acquired by background contextual fear conditioning. However, the results showed no effect of propranolol on contextual fear (Drug $\times$ Session, $F_{1,20}=1.591, p=0.222$, Figure $\left.5 \mathrm{~B}\right)$.

A

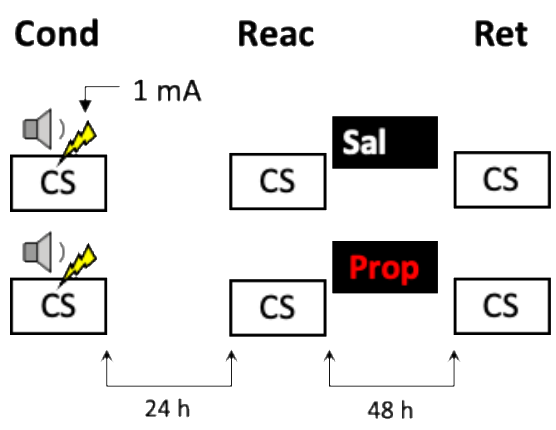

B

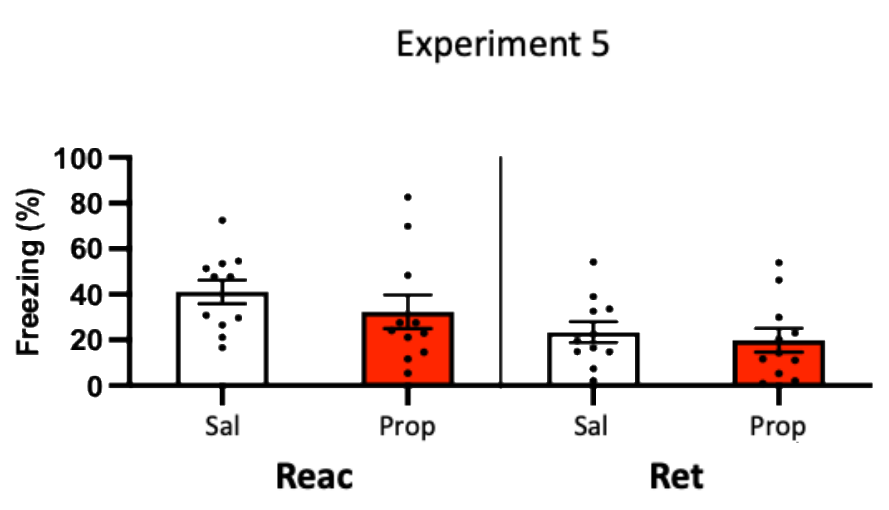

Fig. 5. No effect of propranolol on reactivated memories in a background contextual fear conditioning paradigm. (A) Design of Experiment 5. Mice received one $1 \mathrm{~mA}$ shock during presentation of a tone. One day later, mice received saline $(n=11)$ or propranolol $(n=11)$ after contextual memory reactivation. Forty-eight hours afterwards, retrieval of contextual fear was assessed by exposure to the context. Cond $=$ conditioning, Reac $=$ reactivation, Ret $=$ retrieval, $\mathrm{CS}=$ conditioned stimulus (context), Sal $=$ saline, Prop $=$ propranolol. $($ B $)$ Results of Experiment 5. Average percentage of freezing during memory reactivation is displayed on the left pane of the column chart, and freezing during contextual memory retrieval on the middle and right panes of the column chart (saline in white bars, propranolol in red bars). Error bars represent SEM. Filled circles indicate individual animals.

3.6. Experiment 6. In Experiments 3-5, we aimed to obtain a more pronounced reduction in fear responding by post-reactivation administration of propranolol than in Experiment 2 by studying 
potential ways that could increase the efficacy of propranolol. We did so by altering the volume in which propranolol is injected (Experiment 3) and creating contextual fear memories that could be more sensitive to noradrenergic signaling (Experiments 4-5). Since no amnestic effects were observed, we reasoned that the null findings may not be caused by an inefficacy of propranolol, but rather unsuccessful induction of memory destabilization. To shed light on this idea, we repeated Experiment 2 but now included a condition in which anisomycin was administered, a potent inhibitor of eukaryotic protein synthesis (Barbacid \& Vazquez, 1974) and one of the most widely studied amnestic agents in the reconsolidation literature (e.g., Blundell, Kouser, \& Powell, 2008; Kwak, Choi, Bakes, Lee, \& Kaang, 2012; Lee, Everitt, \& Thomas, 2004; Nader et al., 2000; Parsons, Gafford, Baruch, Riedner, \& Helmstetter, 2006; Rao-Ruiz et al., 2011; Suzuki et al., 2004). No reduction in fear responding by either anisomycin or propranolol was found (Drug $\times$ Session, $F_{2,22}=0.327, p=0.724$, Figure 6B). Furthermore, a follow-up analysis with the collapsed data of the saline and propranolol conditions in Experiment 3 and 6 showed no significant amnesic effects of propranolol $\left(F_{1,42}=0.530, p=0.471\right)$, unlike the pooled data of Experiment 2 in which the same procedure was followed (compare Figures 3B and 6B vs. Figure 2D). In sum, the findings seem to indicate that the lack of post-reactivation amnesia that we observe seems attributable to an ineffectiveness of the memory reactivation session in triggering memory destabilization and hence its subsequent reconsolidation process. 
A

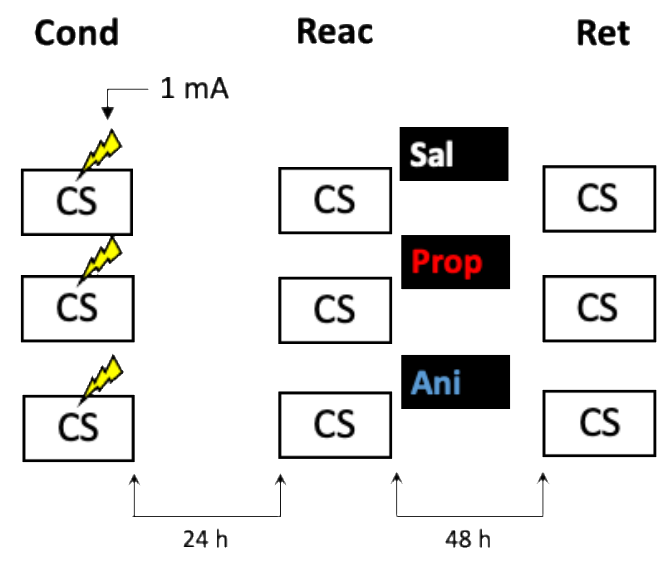

B

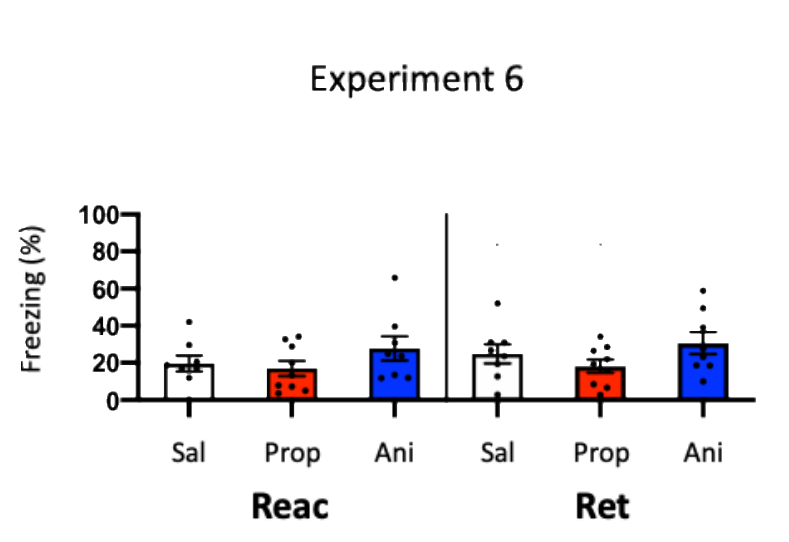

Fig. 6. No effect of propranolol or anisomycin on reactivated memories that were acquired with a single $1 \mathrm{~mA}$ shock during contextual fear conditioning. (A) Design of Experiment 6. Mice received one $1 \mathrm{~mA}$ shock during fear conditioning. One day later, mice received saline $(n=8)$, propranolol $(n=9)$, or anisomycin $(n=8)$ after reactivation. Forty-eight hours afterwards, retrieval of contextual fear was assessed. Cond $=$ conditioning, Reac $=$ reactivation, Ret $=$ retrieval, $\mathrm{CS}=$ conditioned stimulus $($ context $), \mathrm{Sal}=$ saline, Prop $=$ propranolol, Ani $=$ anisomycin. $(\mathbf{B})$ Results of Experiment 6. Average percentage of freezing during memory reactivation is displayed on the left, and freezing during contextual memory retrieval on the right pane of the column chart (saline in white bars, propranolol in red bars, and anisomycin in blue bars). Error bars represent SEM. Filled circles indicate individual animals.

\subsection{Experiment 7. In order to successfully trigger memory destabilization, it is imperative to}

have a proper memory acquired. In most of the experiments conducted so far, freezing levels during

memory reactivation varied greatly, with maximum freezing levels around 60-80\% and minimum

freezing below $10 \%$ within single experiments. Hence, mice may have differred substantially in the

extent to which their fear memories were reactivated and destabilized, such that reconsolidation was

triggered in only a subset of the subjects, leading to small effects at best. In the next experiments, we therefore aimed to test training protocols that would lead to relatively homogenous freezing during reactivation across subjects in the next experiments. As learning by repetition is one of the most classic and widely-known principles of memory (Ebbinghaus, 2013), we administered multiple USs at regular intervals, instead of a single foot shock, as a means to induce robust fear learning in all animals. In Experiment 7, we presented three $0.5 \mathrm{~mA}$ shocks during conditioning, a procedure that has been shown to result in a fear memory that is vulnerable to post-reactivation administration of amnestic agents (Schmidt et al., 2017). We included both propranolol and anisomycin conditions. 
Again, no amnestic effect of propranolol or anisomycin was observed (Drug $\times$ Session, $F_{2,24}=$ $0.205, p=0.816$, Figure 7B). Furthermore, an additional cross-experimental comparison showed that freezing levels during reactivation were significantly higher in the present experiment that involved three $0.5 \mathrm{~mA}$ shocks, than the previous one in which a single $1 \mathrm{~mA}$ shock was administered $\left(t_{50}=-\right.$ 7.161, $p<0.001$, compare Figures 6B and 7B).

A

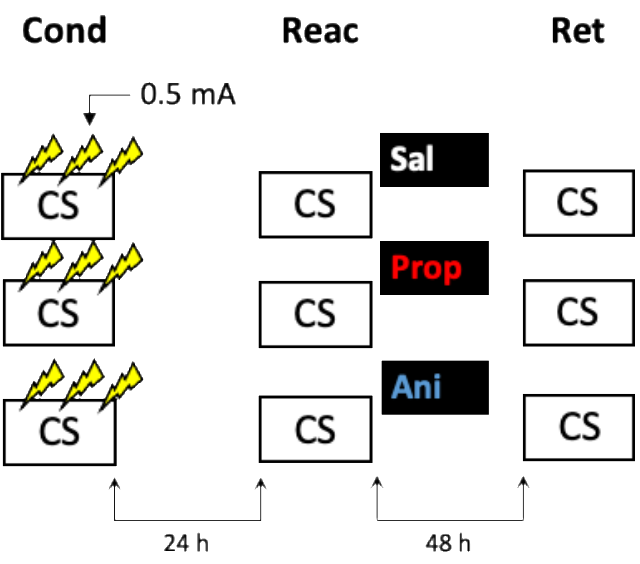

B

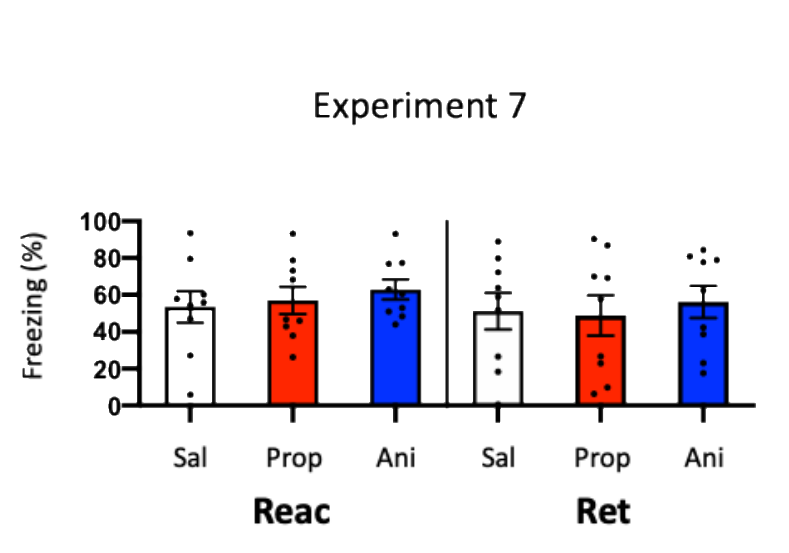

Fig. 7. No effect of propranolol or anisomycin on reactivated memories that were acquired with three $0.5 \mathrm{~mA}$ shocks during contextual fear conditioning. (A) Design of Experiment 7. Mice received three $0.5 \mathrm{~mA}$ shocks during fear conditioning. One day later, mice received saline $(n=9)$, propranolol $(n=9)$, or anisomycin $(n=9)$ after reactivation. Forty-eight hours afterwards, retrieval of contextual fear was assessed. Cond $=$ conditioning, Reac $=$ reactivation, Ret $=$ retrieval, $\mathrm{CS}=$ conditioned stimulus (context), $\mathrm{Sal}=$ saline, $\mathrm{Prop}=$ propranolol, Ani $=$ anisomycin. $(\mathbf{B})$ Results of Experiment 7. Average percentage of freezing during memory reactivation is displayed on the left, and freezing during contextual memory retrieval on the right pane of the column chart (saline in white bars, propranolol in red bars, and anisomycin in blue bars). Error bars represent SEM. Filled circles indicate individual animals.

3.8. Experiment 8. Previous research has shown that increasing the number of administered shocks in contextual fear conditioning leads to higher freezing during subsequent memory retrieval (Poulos et al., 2016). Therefore, it is possible that several USs of a lower intensity results in a stronger fear memory than a single higher intensity US. The freezing levels during reactivation in Experiment 7 (three $0.5 \mathrm{~mA}$ shocks), relative to Experiment 6 (one $1 \mathrm{~mA}$ shock), were enhanced to such a striking extent (Cohen's $d=1.99$ ) that memory strength may have prevented the occurrence of diminished fear responding by administration of amnestic agents upon memory reactivation (Suzuki et al., 2004). In a final attempt to observe post-reactivation amnesia, we aimed to obtain relatively 
homogenous freezing using multiple shocks, but at the same time not induce a fear memory that is much stronger than in the previous experiments that included a single shock. Specifically, we repeated Experiment 7 and lowered the shock intensity to $0.3 \mathrm{~mA}$ (Experiment $8 \mathrm{a}$ ).

Once again, no evidence for a post-reactivation amnestic effect of propranolol or anisomycin was found (Drug $\times$ Session, $F_{2,24}=1.227, p=0.311$, Figure $\left.8 \mathrm{~B}\right)$. When animals that showed freezing levels below 10\% during reactivation were excluded a Drug x Session interaction at trend level was found $\left(F_{2,17}=3.214, p=0.065\right.$, supplementary Figure S1L). Additional independent t-tests showed that freezing during retrieval was lowered in the propranolol condition, relative to the saline condition $\left(t_{11}=2.264, p=0.045\right)$. Surprisingly, this was not the case in the anisomycin condition $\left(t_{11}\right.$ $=0.645, p=0.532$ ). Although a reduction in fear responding by post-reactivation administration of propranolol is in line with earlier reports on the involvement of beta-adrenergic receptors in memory reconsolidation (Johansen et al., 2011), the results of Experiment 8a are hard to reconcile with the presumed protein dependency of this process (Nader et al., 2000). Integration theory as an alternative account, also does not provide an explanation, as there is no indication that propranolol would, but anisomycin would not, induce a state-dependent memory (Gisquet-Verrier \& Riccio, 2018). Furthermore, as this reducing effect of propranolol on freezing levels from reactivation to retrieval was only observed with the specific inclusion criterion of at least $10 \%$ freezing during reactivation, it is uncertain to what extent this finding is robust. We therefore performed a repetition of the experiment (Experiment 8b) in which we aimed to maximize the chance of observing postreactivation amnesia by administration of propranolol. Specifically, we included a condition in which propranolol was injected in the same concentration as in the previous experiment ( $2 \mathrm{mg}$ propranolol/1 $\mathrm{mL}$ saline), and a condition in which half the concentration and double the volume was injected as in 
A

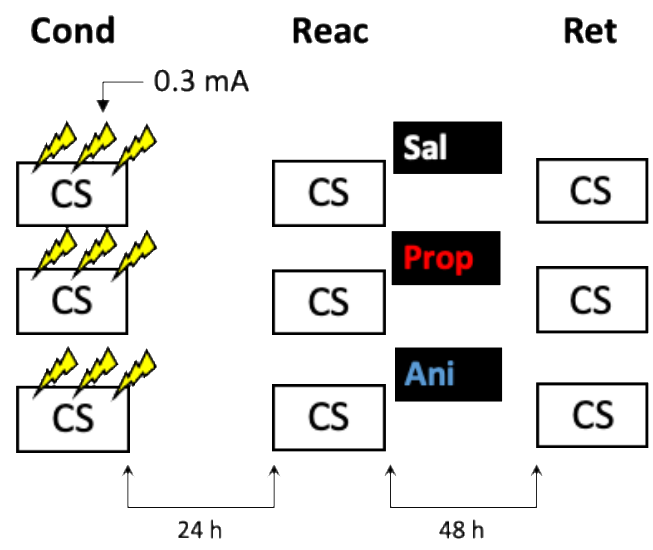

C

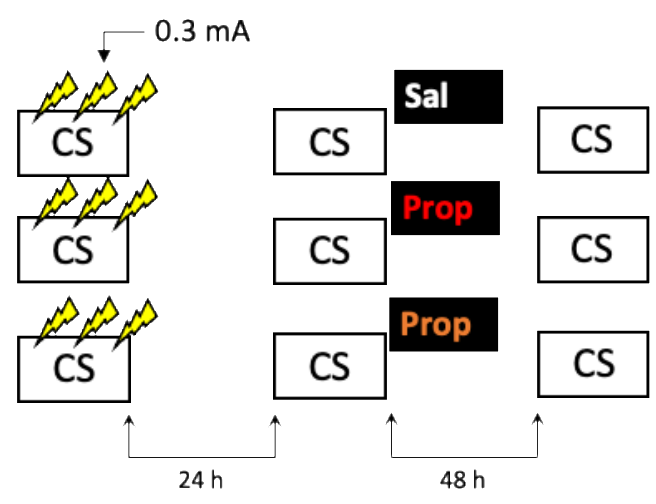

Fig. 8. No effect of propranolol or anisomycin on reactivated memories that were acquired with three $0.3 \mathrm{~mA}$ shocks during contextual fear conditioning. (A) Design of Experiment 8a. Mice received three $0.3 \mathrm{~mA}$ shocks during fear conditioning. One day later, mice received saline $(n=9)$, propranolol $(n=$ $9)$, or anisomycin $(n=9)$ after reactivation. Forty-eight hours afterwards, retrieval of contextual fear was assessed. Cond $=$ conditioning, Reac $=$ reactivation, Ret $=$ retrieval, $\mathrm{CS}=$ conditioned stimulus (context), Sal $=$ saline, Prop = propranolol, Ani = anisomycin. $(\mathbf{B})$ Results of Experiment 8a. Average percentage of freezing during memory reactivation is displayed on the left, and freezing during contextual memory retrieval on the right pane of the column chart (saline in white bars, propranolol in red bars, and anisomycin in blue bars). (C) Design of Experiment 8b. The anisomycin condition was replaced with propranolol dissolved in saline at half the usual ratio and injected in double the volume/body weight $(n=9$ in all conditions). All other procedures were the same as Experiment 8a. (D) Results of Experiment 8b (saline in white bars, propranolol in red bars, and propranolol double volume in orange bars). (E) Collapsed results of Experiment 8a and $8 \mathrm{~b}$ (propranolol in all volumes). Error bars represent SEM. Filled circles indicate individual animals.

Experiment 3 (Villain et al., 2016), such that two different administrations were used that might

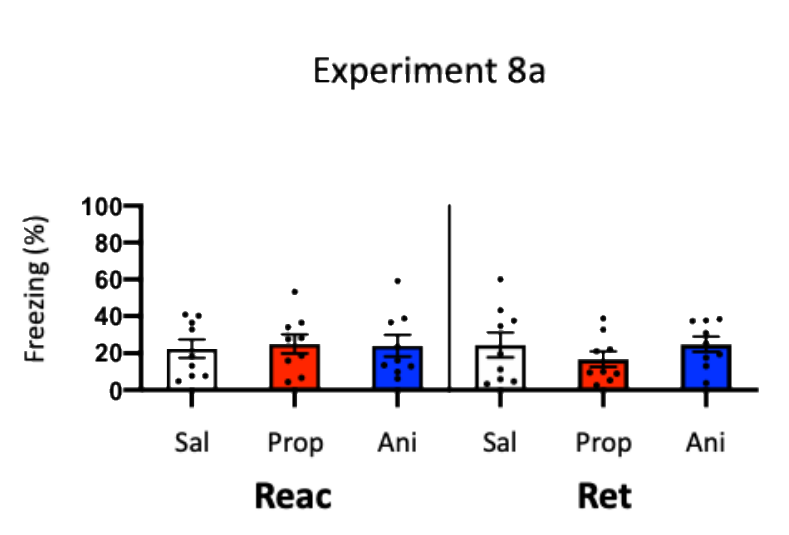

D

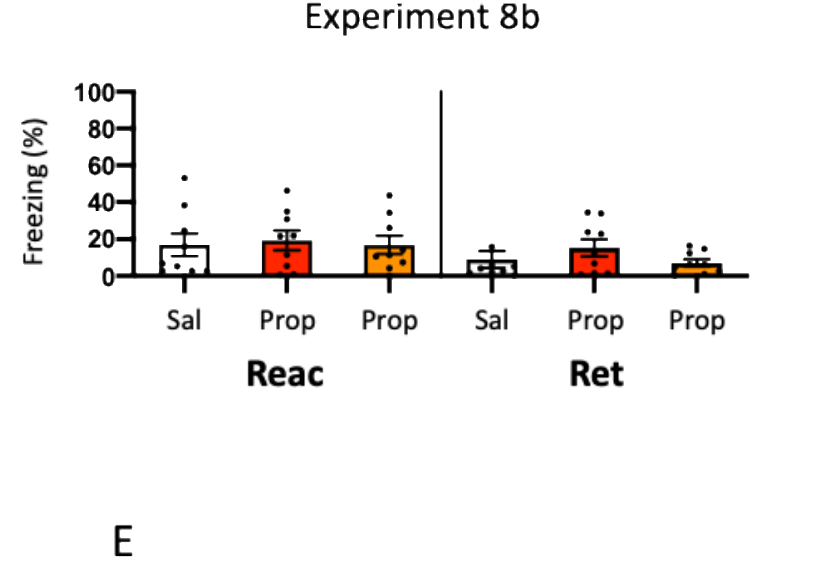

Experiment 8 (collapsed)

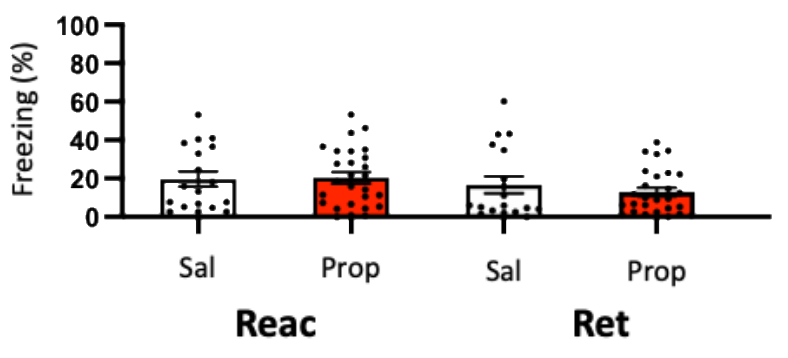
result in diminished fear responding. 
No reduced fear responding by administration of propranolol was observed when all mice were included (Drug $\times$ Session, $F_{2,24}=0.737, p=0.489$, Figure 8D), or when mice that showed freezing levels below $10 \%$ were excluded (Drug $\times$ Session, $F_{2,13}=1.632, p=0.233$, Figure S1M). Since in experiment $8 \mathrm{~b}$ a large number of mice showed freezing levels below $10 \%$, there was low statistical power to replicate the propranolol-induced reduction in fear responding that was observed in Experiment $8 \mathrm{a}(1-\beta=0.40)$. We, therefore, also analyzed collapsed data of Experiment $8 \mathrm{a}$ and $8 \mathrm{~b}$. Both with $\left(F_{1,43}=1.261, p=0.268\right.$, Figure $\left.8 \mathrm{E}\right)$ and without $\left(F_{1,27}=2.249, p=0.145\right.$, Figure $\left.\mathrm{S} 1 \mathrm{~N}\right)$ inclusion of mice that showed freezing levels lower than $10 \%$ during reactivation, no effect of propranolol (in any volume) was found, suggesting that the post-reactivation amnesia as observed in Experiment 8a was not robust. Furthermore, a cross-experimental comparison (Experiment 8 vs. Experiment 6, compare Figures 8E and 6B) showed that administering multiple shocks, instead of a single shock, did not lead to more homogenous freezing during reactivation (Levene's test, $F_{1,68}=$ $1.313, p=0.256$ ), which was the aim of the last two experiments. Thus, as the different behavioral protocols involving single or multiple foot shocks of varying intensities did not provide a stable paradigm to study post-reactivation amnesia, and no clear indications of how to improve the protocol seemed to be at hand, no further experiments were carried out.

\section{Discussion}

We performed a series of experiments to study the effects of post-reactivation, systemic administration of propranolol on contextual fear memory in mice. The eventual purpose of this study was to elucidate the neural mechanisms by which propranolol has been shown to interfere with contextual fear memories (Abrari et al., 2008; Liu et al., 2015), and test ways to overcome boundary conditions that potentially complicate application of post-reactivation amnesia in clinical practice (Kindt, 2014). However, in a total of 11 experiments (including replicates) that involved different 
fear acquisition procedures and drugs (i.e., not only propranolol, but also anisomycin), our attempts to observe robust amnesic effects were largely unsuccessful. At best, slight freezing reductions were found in a minority of the experiments.

Specifically, only when the data of Experiments $2 \mathrm{a}$ and $2 \mathrm{~b}$ were collapsed, a marginally significant effect of post-reactivation administration of propranolol on freezing was observed. Note that in these experiments we attempted to create stronger fear memories relative to Experiment 1 by raising the intensity of the delivered foot shock. It is remarkable that we indeed found an indication of reduced fear responding in the propranolol condition in Experiment 2, even though the freezing levels during memory reactivation were not significantly higher in this experiment than in Experiment 1. As it may seem unlikely that experiments with comparable levels of freezing during memory reactivation sometimes do (Experiments 2), and sometimes do not (Experiment 1) show pharmacologically-induced amnesia after memory reactivation, one could question whether the finding in Experiment 2 is a true positive. Previous research has indicated that the expression of fear during memory reactivation is not an accurate index of memories entering a labile state. Emotional memory expression during memory reactivation is not a necessary, nor a sufficient condition for the occurrence of post-reactivation amnesia (see Faliagkas et al., 2018 for a review). Despite the freezing levels in Experiments 1 and 2 being similar, the memory processes that were triggered by reactivation were therefore not necessarily identical. Nevertheless, the effect in Experiments 2 was considerably smaller than in earlier demonstrations of reduced fear responding by administration of propranolol after memory reactivation (e.g., Przybyslawski et al., 1999; Dębiec \& Ledoux, 2004; Liu et al., 2015), and the small effect did not replicate in two additional experiments (Experiments 3 and 6). The minor amnestic effect also did not translate to a difference in neural activity between experimental groups. All in all, although the results of Experiments 2 were perhaps not a chance finding, the observed reduction in fear responding by post-reactivation administration of propranolol was clearly not robust either. 
Why were our attempts to develop a paradigm that would show consistent effects of amnestic agents on reactivated memories unsuccessful, given that we tried a wide variety of procedures? A few factors could be considered. The null findings do not seem to be attributable to a lack of power. The number of mice that were included in each experiment were comparable to previous studies in which large effects were observed (e.g., Przybyslawski et al., 1999; Dębiec \& Ledoux, 2004; Liu et al., 2015). We also did not find robust effects when the data of similar experiments (Experiment 1 and 2, Experiment 3 and 4, Experiment 3 and 6) or all experiments performed within the same research instrument (TSE, or Med Associates, see supplementary results) were collapsed. Furthermore, a general inefficiency of propranolol in interfering with fear memories does not seem to be a plausible explanation either. We administered propranolol in the same dose as in previous experiments that have shown robust post-reactivation amnesia in both rats (Dębiec \& Ledoux, 2004; Przybyslawski et al., 1999) and mice (Liu et al., 2015; Villain et al., 2016), and in two different volumes of saline, of which one has been used earlier in mice (Villain et al., 2016). We also included anisomycin in several experiments, which in previous research has induced amnestic effects across various protocols. For example, reduced fear responding by local or systemic administrations of anisomycin before or after reactivation of contextual or cued fear have all been observed (e.g., Blundell et al., 2008; Kwak et al., 2012; Lee et al., 2004; Nader et al., 2000; Parsons et al., 2006; Rao-Ruiz et al., 2011; Suzuki et al., 2004).

A more likely explanation for the null findings may be that the procedures did not (consistently) trigger the memories to enter a state in which it becomes receptive to change. Previous research has identified several boundary conditions for reactivation-dependent memory plasticity (Lee, 2009; Pedreira et al., 2004). For example, both memories that are reactivated rather shortly or lengthily (Merlo et al., 2014; Sevenster et al., 2012, 2014), in relation to their strength (Suzuki et al., 2004), may not undergo weakening by administration of amnestic agents. At first sight, it seems like we have largely ruled out this explanation, since we have varied the fear conditioning procedures 
across experiments to induce memories of different strengths. However, the experiments showed highly variable levels of freezing during memory reactivation, ranging from lower than 5\% to higher than $85 \%$ within a single condition in the most extreme cases. It therefore remains possible that memories became vulnerable to the administration of propranolol or anicomycin in only a small number of mice (although in some previous studies post-reactivation amnesia was observed with somewhat similar spread of freezing during reactivation, Dȩbiec et al., 2002; Rao-Ruiz et al., 2011). Since boundary conditions can complicate falsifiability of any phenomenon (Elsey \& Kindt, 2017), it is important to test such explanations before final conclusions are drawn. For this reason, we have performed experiments (Experiment 7-8) that were specifically aimed at reducing the spread of freezing during memory reactivation. These experiments were unsuccessful in this regard. Whether inconsistency of memory destabilization is the cause of the present (or other) null findings, at this point, remains an open question.

Another possibility to consider, although speculative, is that the inbred experimental animal strain used by us has undergone changes over the past years, such that procedures that used to be effective no longer trigger a state of memory plasticity (Schroyens et al., 2019). It is remarkable in this light that Experiment 1 was procedurally identical to an earlier study (Rao-Ruiz et al., 2011) in many respects (animal strain, supplier, lab space, behavioral system, training protocol and length of memory reactivation and retrieval tests), but now showed much lower freezing levels during reactivation. This discrepancy might be explained by previous research showing that inbred mouse strains are not always isogenic. Factors such as genetic drift, spontaneous mutations and epigenetic changes may all influence the behavior of experimental animals (Chebib, Jackson, López-Cortegano, Tautz, \& Keightley, 2021; Loos et al., 2015; Oey, Isbel, Hickey, Ebaid, \& Whitelaw, 2015; Stiedl et al., 1999). We tried to control for this variability by ordering animals from a well-established breeder (Charles River laboratories, France) that has robust genetic monitoring programs in place. However, although the commonly used genetic screens of commercial breeders minimize cross-strain 
contamination, they are less successful at identifying spontaneous mutations and epigenetic changes that may drive behavioral variability without presenting as overt phenotypes. Isogenicity within the inbred strain can therefore not be assumed (Chebib et al., 2021).

Besides genetic variability, environmental factors may also contribute to the limited replicability of reactivation-induced amnesia that we observe. Factors such as breeding conditions, colony maintenance, maternal care or transportation, which are all beyond the researchers' sight or control, might impact the success of downstream experimental manipulations. For example, early life stress, which could potentially occur or vary among experimental animals before their arrival at the research site, has been shown to have an influence on both fear learning (Kosten, Kim, \& Lee, 2012) and memory plasticity (Couto-Pereira et al., 2019; Villain, Benkahoul, Birmes, Ferry, \& Roullet, 2018). Variations in maternal care and colony maintenance can also affect adult behavioral outcomes and stress reactivity of C57B16/J mice (Pedersen, Vadlamudi, Boccia, \& Moy, 2011).

A possibility to consider in light of the discrepant freezing levels across studies, is that genetic factors, environmental factors, or an interaction thereof, which have been shown to contribute to within-strain variability (Loos et al., 2015), result in a change in risk assessment of aversive stimuli and a choice in downstream defensive strategies by experimental animals. Aversive learning can contain explicit and implicit memory systems that are not readily dissociable in rodents (Wotjak, 2019). This is attributable to a lack of adequate readouts that can distinguish between behavioral outcomes driven by associative and non-associative components of the fear circuit (Wotjak, 2019). Automated fear conditioning systems, like the ones used in this study, measure freezing as the sole behavioral output of fear. However, in addition to vegetative (e.g. changes in heart rate, respiration) and hormonal (e.g. changes in stress hormone levels) responses, rodents can use risk assessment to choose an appropriate behavioral defensive response to threatening stimuli that they encounter (Blanchard, Griebel, Pobbe, \& Blanchard, 2011). This analytic function enables animals to choose a strategy most likely to succeed in a given situation and include freezing, flight/escape, startle, 
burying, ultrasonic vocalizations, or a combination thereof (Wotjak, 2019). A switch in defensive strategy, such as active 'flight' responding instead of a cessation of movement, would result in generally lower freezing levels that could potentially affect behavioral readouts and confound the interpretation of the amnestic effects of the applied interventions. The particularly low freezing in Experiment 4 in which the experimental procedures were executed in the animals' dark phase suggests that mice can indeed use different of such defensive strategies in response to the same aversive stimuli. Similar to our arguments concerning variability in the strength of memory reactivation, the influence of genetic and environmental factors might prove to be difficult to control, and in that case remain unfalsifiable. However, being able to breed and maintain inbred colonies inhouse or change to paradigms that call upon more active defensive responses, such as inhibitory avoidance, could shed light on some of these ideas.

Note that in the present series of experiments, no direct replication attempts of earlier studies have been performed, which has been done in some experiments of Schroyens et al., 2019. The findings presented here do not suggest that previously reported results are false positives. Due to drastic changes in the animals' behavior as discussed above, the overarching approach towards a paradigm with consistent effects was to gradually build on the outcomes of individual experiments in the series. Although we have not been successful in doing so, we believe that this might be the most fruitful way forward. Apart from replication studies showing that seemingly basic manipulations do not always have consistent outcomes, systematically varying single factors such as the strength and/or the number of the aversive stimulus presentations, as well the duration and time-point of reactivation, could contribute to regaining control over reactivation-dependent manipulation of memory in a step-by-step fashion. Our findings add to this endeavor, specifically in relation to contextual fear memories in mice.

Apart from freezing, we did not observe effects of post-reactivation administration of propranolol at a neural level either, which is reminiscent of a recent study by Leal Santos et al. 
(2021). These authors demonstrated that propranolol only has an acute effect on contextual memory expression and DG memory trace cells when delivered immediately prior to memory reactivation. No long-term effect on memory retrieval after drug washout (i.e., 24 h later) was observed. The authors like us - observed no effect of post-reactivation administration of propranolol on subsequent memory retrieval, while using a different mouse strain (129S6/SvEv) and conditioning protocol (4 x $0.75 \mathrm{~mA}$ foot shocks) than in the present study. Together, our data and those of Leal Santos et al. (2021) thus seem to indicate that propranolol does not have lasting effects on neural activity in the hippocampal DG.

In conclusion, our findings show that inducing post-reactivation amnesia is not as straightforward as the overall state of the published literature on reconsolidation suggests, yet align with a recent report of null findings in rats (Schroyens et al., 2019). As we used a different species, laboratory equipment, behavioral protocols, drugs, and included larger samples in each experiment than in Schroyens et al., 2019, the findings show that obstacles in observing robust reactivationdependent reductions in fear responding are not uniquely related to the parameters varied by particular research groups. Clearly, the present findings do not rule out the existence of postreactivation amnesia. They do underscore the elusive nature of this phenomenon and emphasize the need for better control over and understanding of the intrinsic and extrinsic factors that may govern it.

\section{Author contributions}

Wouter Cox: Conceptualization, Formal Analysis, Investigation, Data Curation, Writing- Original Draft Preparation, Visualization. Leonidas Faliagkas: Conceptualization, Formal Analysis, Investigation, Data Curation. Rolinka van der Loo: Investigation. Sabine Spijker: Conceptualization, Writing- Reviewing and Editing, Funding Acquisition. Merel Kindt: 
Conceptualization, Writing- Reviewing and Editing. Priyanka Rao-Ruiz: Conceptualization,

Methodology, Formal Analysis, Investigation, Resources, Writing- Reviewing and Editing,

Visualization, Supervision, Project Administration, Funding Acquisition.

\section{Funding}

Wouter Cox is supported by a Research Talent grant from the Netherlands Organization for Scientific Research (406-16-557). Leonidas Faliagkas and Sabine Spijker are supported by NWO VICI grant (ALW-Vici 016.150.673/865.14.002). Merel Kindt is supported by an ERC Advanced Grant (743263). Priyanka Rao-Ruiz is supported by the Amsterdam Brain and Mind project, and the ZonMW TOP (\#91215030).

\section{Conflict of interest}

Merel Kindt has co-founded an outpatient clinic (Kindt Clinics), which offers reconsolidation-based treatments for anxiety disorders. The authors declare no conflicts of interest.

\section{References}

Abrari, K., Rashidy-Pour, A., Semnanian, S., \& Fathollahi, Y. (2008). Administration of corticosterone after memory reactivation disrupts subsequent retrieval of a contextual conditioned fear memory: Dependence upon training intensity. Neurobiology of Learning and Memory, 89, 178-184.

Alberini, C. M. (2005). Mechanisms of memory stabilization: Are consolidation and reconsolidation similar or distinct processes? Trends in Neurosciences, 28, 51-56. 
Anagnostaras, S. G., Wood, S. C., Shuman, T., Cai, D. J., LeDuc, A. D., Zurn, K. R., ... Herrera, G. M. (2010). Automated assessment of Pavlovian conditioned freezing and shock reactivity in mice using the VideoFreeze system. Frontiers in Behavioral Neuroscience, 4, 158.

Aston-Jones, G., \& Bloom, F. E. (1981). Activity of norepinephrine-containing locus coeruleus neurons in behaving rats anticipates fluctuations in the sleep-waking cycle. The Journal of Neuroscience, 1, 876-886.

Barbacid, M., \& Vazquez, D. (1974). [3H]anisomycin binding to eukaryotic ribosomes. Journal of Molecular Biology, 84, 603-623.

Blanchard, D. C., Griebel, G., Pobbe, R., \& Blanchard, R. J. (2011). Risk assessment as an evolved threat detection and analysis process. Neuroscience and Biobehavioral Reviews, 35, 991-998.

Blundell, J., Kouser, M., \& Powell, C. M. (2008). Systemic inhibition of mammalian target of rapamycin inhibits fear memory reconsolidation. Neurobiology of Learning and Memory, 90, 28-35.

Bos, M. G. N., Beckers, T., \& Kindt, M. (2014). Noradrenergic blockade of memory reconsolidation: A failure to reduce conditioned fear responding. Frontiers in Behavioral Neuroscience, 8, 412.

Bouton, M. E. (2002). Context, ambiguity, and unlearning: Sources of relapse after behavioral extinction. Biological Psychiatry, 52, 976-986.

Brunet, A., Orr, S. P., Tremblay, J., Robertson, K., Nader, K., \& Pitman, R. K. (2008). Effect of postretrieval propranolol on psychophysiologic responding during subsequent script-driven traumatic imagery in post-traumatic stress disorder. Journal of Psychiatric Research, 42, 503506. 
Brunet, A., Poundja, J., Tremblay, J., Bui, É., Thomas, É., Orr, S. P., .. Pitman, R. K. (2011).

Trauma reactivation under the influence of propranolol decreases posttraumatic stress symptoms and disorder: 3 open-label trials. Journal of Clinical Psychopharmacology, 31, 547-550.

Brunet, A., Saumier, D., Liu, A., Streiner, D. L., Tremblay, J., \& Pitman, R. K. (2018). Reduction of PTSD symptoms with pre-reactivation propranolol therapy: A randomized controlled trial. American Journal of Psychiatry, 175, 427-433.

Bustos, S. G., Maldonado, H., \& Molina, V. A. (2006). Midazolam disrupts fear memory reconsolidation. Neuroscience, 139, 831-842.

Bustos, S. G., Maldonado, H., \& Molina, V. A. (2009). Disruptive effect of midazolam on fear memory reconsolidation: Decisive influence of reactivation time span and memory age. Neuropsychopharmacology, 34, 446-457.

Chalkia, A., Weermeijer, J., Van Oudenhove, L., \& Beckers, T. (2019). Acute but not permanent effects of propranolol on fear memory expression in humans. Frontiers in Human Neuroscience, 13,51 .

Chebib, J., Jackson, B. C., López-Cortegano, E., Tautz, D., \& Keightley, P. D. (2021). Inbred lab mice are not isogenic: Genetic variation within inbred strains used to infer the mutation rate per nucleotide site. Heredity, 126, 107-116.

Cirelli, C., \& Tononi, G. (2000). Differential expression of plasticity-related genes in waking and sleep and their regulation by the noradrenergic system. The Journal of Neuroscience, 20, 91879194.

Couto-Pereira, N. de S., Lampert, C., Vieira, A. dos S., Lazzaretti, C., Kincheski, G. C., Espejo, P. J., ... Dalmaz, C. (2019). Resilience and vulnerability to trauma: Early life interventions modulate 
aversive memory reconsolidation in the dorsal hippocampus. Frontiers in Molecular Neuroscience, 12, 134.

Cruickshank, J. M. (1980). The clinical importance of cardioselectivity and lipophilicity in beta blockers. American Heart Journal, 100, 160-178.

Dębiec, J., \& Ledoux, J. E. (2004). Disruption of reconsolidation but not consolidation of auditory fear conditioning by noradrenergic blockade in the amygdala. Neuroscience, 129, 267-272.

Dębiec, J., LeDoux, J. E., \& Nader, K. (2002). Cellular and systems reconsolidation in the hippocampus. Neuron, 36, 527-538.

Denny, C. A., Kheirbek, M. A., Alba, E. L., Tanaka, K. F., Brachman, R. A., Laughman, K. B., ... Hen, R. (2014). Hippocampal memory traces are differentially modulated by experience, time, and adult neurogenesis. Neuron, 83, 189-201.

Duvarci, S., \& Nader, K. (2004). Characterization of fear memory reconsolidation. The Journal of Neuroscience, 24, 9269-9275.

Ebbinghaus, H. (2013). Memory: A contribution to experimental psychology. Annals Classics, 20, $155-156$.

Eisenberg, M., \& Dudai, Y. (2004). Reconsolidation of fresh, remote, and extinguished fear memory in medaka: Old fears don't die. European Journal of Neuroscience, 20, 3397-3403.

Elsey, J. W. B., Filmer, A. I., Galvin, H. R., Kurath, J. D., Vossoughi, L., Thomander, L. S., ... Kindt, M. (2020). Reconsolidation-based treatment for fear of public speaking: A systematic pilot study using propranolol. Translational Psychiatry, 10, 179.

Elsey, J. W. B., \& Kindt, M. (2017). Breaking boundaries: Optimizing reconsolidation-based 
interventions for strong and old memories. Learning and Memory, 24, 472-479.

Elsey, J. W. B., van Ast, V. A., \& Kindt, M. (2018). Human memory reconsolidation: A guiding framework and critical review of the evidence. Psychological Bulletin, 144, 797-848.

Faliagkas, L., Rao-Ruiz, P., \& Kindt, M. (2018). Emotional memory expression is misleading:

Delineating transitions between memory processes. Current Opinion in Behavioral Sciences, 19, $116-122$.

Gallagher, M., Kapp, B. S., Musty, R. E., \& Driscoll, P. A. (1977). Memory formation: Evidence for a specific neurochemical system in the amygdala. Science, 198, 423-425.

Gelinas, J. N., \& Nguyen, P. V. (2005). B-Adrenergic receptor activation facilitates induction of a protein synthesis-dependent late phase of long-term potentiation. The Journal of Neuroscience, 25, 3294-3303.

Gisquet-Verrier, P., \& Riccio, D. C. (2018). Memory integration: An alternative to the consolidation/reconsolidation hypothesis. Progress in Neurobiology, 171, 15-31.

Giustino, T. F., \& Maren, S. (2018). Noradrenergic modulation of fear conditioning and extinction. Frontiers in Behavioral Neuroscience, 12, 43.

Gouty-Colomer, L. A., Hosseini, B., Marcelo, I. M., Schreiber, J., Slump, D. E., Yamaguchi, S., ... Kushner, S. A. (2016). Arc expression identifies the lateral amygdala fear memory trace. Molecular Psychiatry, 21, 364-375.

Ji, J.-Z., Wang, X.-M., \& Li, B.-M. (2003). Deficit in long-term contextual fear memory induced by blockade of $\beta$-adrenoceptors in hippocampal CA1 region. European Journal of Neuroscience, 17, 1947-1952. 
Joëls, M., Fernandez, G., \& Roozendaal, B. (2011). Stress and emotional memory: A matter of timing. Trends in Cognitive Sciences, 15, 280-288.

Johansen, J. P., Cain, C. K., Ostroff, L. E., \& Ledoux, J. E. (2011). Molecular mechanisms of fear learning and memory. Cell, 147, 509-524.

Kindt, M. (2014). A behavioural neuroscience perspective on the aetiology and treatment of anxiety disorders. Behaviour Research and Therapy, 62, 24-36.

Kindt, M., \& Soeter, M. (2018). Pharmacologically induced amnesia for learned fear is time and sleep dependent. Nature Communications, 9, 1316.

Kindt, M., Soeter, M., \& Vervliet, B. (2009). Beyond extinction: Erasing human fear responses and preventing the return of fear. Nature Neuroscience, 12, 256-258.

Kindt, M., \& van Emmerik, A. (2016). New avenues for treating emotional memory disorders: Towards a reconsolidation intervention for posttraumatic stress disorder. Therapeutic Advances in Psychopharmacology, 6, 283-295.

Kosten, T. A., Kim, J. J., \& Lee, H. J. (2012). Early life manipulations alter learning and memory in rats. Neuroscience and Biobehavioral Reviews, 36, 1985-2006.

Kwak, C., Choi, J.-H., Bakes, J. T., Lee, K., \& Kaang, B. K. (2012). Effect of intensity of unconditional stimulus on reconsolidation of contextual fear memory. Korean Journal of Physiology and Pharmacology, 16, 293-296.

LaLumiere, R. T., Buen, T.-V., \& McGaugh, J. L. (2003). Post-training intra-basolateral amygdala infusions of norepinephrine enhance consolidation of memory for contextual fear conditioning. The Journal of Neuroscience, 23, 6754-6758. 
Lattal, K. M., \& Abel, T. (2004). Behavioral impairments caused by injections of the protein synthesis inhibitor anisomycin after contextual retrieval reverse with time. Proceedings of the National Academy of Sciences of the United States of America, 101, 4667-4672.

Leal Santos, S., Stackmann, M., Muñoz Zamora, A., Mastrodonato, A., De Landri, A. V., Vaughan, N., ... Denny, C. A. (2021). Propranolol decreases fear expression by modulating fear memory traces. Biological Psychiatry, 89, 1150-1161.

Lee, J. L. C. (2009). Reconsolidation: Maintaining memory relevance. Trends in Neurosciences, 32 , 413-420.

Lee, J. L. C., Everitt, B. J., \& Thomas, K. L. (2004). Independent cellular processes for hippocampal memory consolidation and reconsolidation. Science, 304, 839-843.

Liang, K. C., Juler, R. G., \& McGaugh, J. L. (1986). Modulating effects of posttraining epinephrine on memory: Involvement of the amygdala noradrenergic system. Brain Research, 368, 125-133.

Liu, X., Ma, L., Li, H. B., Huang, B., Li, Y. X., Tao, Y. Z., \& Ma, L. (2015). $\beta$-Arrestin-biased signaling mediates memory reconsolidation. Proceedings of the National Academy of Sciences of the United States of America, 112, E2847.

Liu, X., Ramirez, S., Pang, P. T., Puryear, C. B., Govindarajan, A., Deisseroth, K., \& Tonegawa, S. (2012). Optogenetic stimulation of a hippocampal engram activates fear memory recall. Nature, $484,381-385$.

Loos, M., Koopmans, B., Aarts, E., Maroteaux, G., van der Sluis, S., Neuro-BSIK Mouse Phenomics Consortium, ... Smit, A. B. (2015). Within-strain variation in behavior differs consistently between common inbred strains of mice. Mammalian Genome, 26, 348-354. 
McGaugh, J. L. (2002). Memory consolidation and the amygdala: A systems perspective. Trends in Neurosciences, 25, 456-461.

McGaugh, J. L. (2015). Consolidating memories. Annual Review of Psychology, 66, 1-24.

McGaugh, J. L. (2018). Emotional arousal regulation of memory consolidation. Current Opinion in Behavioral Sciences, 19, 55-60.

Merlo, E., Milton, A. L., Goozee, Z. Y., Theobald, D. E., \& Everitt, B. J. (2014). Reconsolidation and extinction are dissociable and mutually exclusive processes: Behavioral and molecular evidence. The Journal of Neuroscience, 34, 2422-2431.

Misanin, J. R., Miller, R. R., \& Lewis, D. J. (1968). Retrograde amnesia produced by electroconvulsive shock after reactivation of a consolidated memory trace. Science, 160, 554555.

Muravieva, E. V., \& Alberini, C. M. (2010). Limited efficacy of propranolol on the reconsolidation of fear memories. Learning and Memory, 17, 306-313.

Nader, K., Schafe, G. E., \& Le Doux, J. E. (2000). Fear memories require protein synthesis in the amygdala for reconsolidation after retrieval. Nature, 406, 722-726.

Oey, H., Isbel, L., Hickey, P., Ebaid, B., \& Whitelaw, E. (2015). Genetic and epigenetic variation among inbred mouse littermates: Identification of inter-individual differentially methylated regions. Epigenetics \& Chromatin, 8, 54.

Parsons, R. G., Gafford, G. M., Baruch, D. E., Riedner, B. A., \& Helmstetter, F. J. (2006). Long-term stability of fear memory depends on the synthesis of protein but not mRNA in the amygdala. European Journal of Neuroscience, 23, 1853-1859. 
Pedersen, C. A., Vadlamudi, S., Boccia, M. L., \& Moy, S. S. (2011). Variations in maternal behavior in C57BL/6J mice: Behavioral comparisons between adult offspring of high and low pup-licking mothers. Frontiers in Psychiatry, 2, 42.

Pedreira, M. E., Pérez-Cuesta, L. M., \& Maldonado, H. (2004). Mismatch between what is expected and what actually occurs triggers memory reconsolidation or extinction. Learning and Memory, $11,579-585$.

Poulos, A. M., Mehta, N., Lu, B., Amir, D., Livingston, B., Santarelli, A., ... Fanselow, M. S. (2016). Conditioning- and time-dependent increases in context fear and generalization. Learning and Memory, 23, 379-385.

Przybyslawski, J., Roullet, P., \& Sara, S. J. (1999). Attenuation of emotional and nonemotional memories after their reactivation: Role of $\beta$ adrenergic receptors. The Journal of Neuroscience, $19,6623-6628$.

Rao-Ruiz, P., Couey, J. J., Marcelo, I. M., Bouwkamp, C. G., Slump, D. E., Matos, M. R., ... Kushner, S. A. (2019). Engram-specific transcriptome profiling of contextual memory consolidation. Nature Communications, 10, 2232.

Rao-Ruiz, P., Rotaru, D. C., Van Der Loo, R. J., Mansvelder, H. D., Stiedl, O., Smit, A. B., \& Spijker, S. (2011). Retrieval-specific endocytosis of GluA2-AMPARs underlies adaptive reconsolidation of contextual fear. Nature Neuroscience, 14, 1302-1308.

Roozendaal, B., \& Hermans, E. J. (2017). Norepinephrine effects on the encoding and consolidation of emotional memory: Improving synergy between animal and human studies. Current Opinion in Behavioral Sciences, 14, 115-122.

Roozendaal, B., McEwen, B. S., \& Chattarji, S. (2009). Stress, memory and the amygdala. Nature 
Reviews Neuroscience, 10, 423-433.

Roullet, P., Vaiva, G., Véry, E., Bourcier, A., Yrondi, A., Dupuch, L., .. Birmes, P. (2021).

Traumatic memory reactivation with or without propranolol for PTSD and comorbid MD symptoms: A randomised clinical trial. Neuropsychopharmacology, 46, 1643-1649.

Schmidt, S. D., Furini, C. R. G., Zinn, C. G., Cavalcante, L. E., Ferreira, F. F., Behling, J. A. K., ... Izquierdo, I. (2017). Modulation of the consolidation and reconsolidation of fear memory by three different serotonin receptors in hippocampus. Neurobiology of Learning and Memory, 142, $48-54$.

Schroyens, N., Alfei, J. M., Schnell, A. E., Luyten, L., \& Beckers, T. (2019). Limited replicability of drug-induced amnesia after contextual fear memory retrieval in rats. Neurobiology of Learning and Memory, 166, 107105.

Schroyens, N., Beckers, T., \& Kindt, M. (2017). In search for boundary conditions of reconsolidation: A failure of fear memory interference. Frontiers in Behavioral Neuroscience, $11,65$.

Sevenster, D., Beckers, T., \& Kindt, M. (2012). Retrieval per se is not sufficient to trigger reconsolidation of human fear memory. Neurobiology of Learning and Memory, 97, 338-345.

Sevenster, D., Beckers, T., \& Kindt, M. (2013). Prediction error governs pharmacologically induced amnesia for learned fear. Science, 339, 830-833.

Sevenster, D., Beckers, T., \& Kindt, M. (2014). Prediction error demarcates the transition from retrieval, to reconsolidation, to new learning. Learning \& Memory, 21, 580-584.

Soeter, M., \& Kindt, M. (2010). Dissociating response systems: Erasing fear from memory. 
Neurobiology of Learning and Memory, 94, 30-41.

Soeter, M., \& Kindt, M. (2011). Disrupting reconsolidation: Pharmacological and behavioral manipulations. Learning and Memory, 18, 357-366.

Soeter, M., \& Kindt, M. (2012a). Erasing fear for an imagined threat event. Psychoneuroendocrinology, 37, 1769-1779.

Soeter, M., \& Kindt, M. (2012b). Stimulation of the noradrenergic system during memory formation impairs extinction learning but not the disruption of reconsolidation.

Neuropsychopharmacology, 37, 1204-1215.

Soeter, M., \& Kindt, M. (2015a). An abrupt transformation of phobic behavior after a post-retrieval amnesic agent. Biological Psychiatry, 78, 880-886.

Soeter, M., \& Kindt, M. (2015b). Retrieval cues that trigger reconsolidation of associative fear memory are not necessarily an exact replica of the original learning experience. Frontiers in Behavioral Neuroscience, 9, 122.

Stiedl, O., Radulovic, J., Lohmann, R., Birkenfeld, K., Palve, M., Kammermeier, J., ... Spiess, J. (1999). Strain and substrain differences in context- and tone-dependent fear conditioning of inbred mice. Behavioural Brain Research, 104, 1-12.

Suzuki, A., Josselyn, S. A., Frankland, P. W., Masushige, S., Silva, A. J., \& Kida, S. (2004). Memory reconsolidation and extinction have distinct temporal and biochemical signatures. The Journal of Neuroscience, 24, 4787-4795.

Taherian, F., Vafaei, A. A., Vaezi, G. H., Eskandarian, S., Kashef, A., \& Rashidy-Pour, A. (2014). Propranolol-induced impairment of contextual fear memory reconsolidation in rats: A similar 
effect on weak and strong recent and remote memories. Basic and Clinical Neuroscience, 5, 231-239.

Thonberg, H., Fredriksson, J. M., Nedergaard, J., \& Cannon, B. (2002). A novel pathway for adrenergic stimulation of cAMP-response-element-binding protein (CREB) phosphorylation: Mediation via $\alpha 1$-adrenoceptors and protein kinase C activation. Biochemical Journal, 364, 7379.

Trifilieff, P., Herry, C., Vanhoutte, P., Caboche, J., Desmedt, A., Riedel, G., .. Micheau, J. (2006). Foreground contextual fear memory consolidation requires two independent phases of hippocampal ERK/CREB activation. Learning and Memory, 13, 349-358.

Végh, M. J., Heldring, C. M., Kamphuis, W., Hijazi, S., Timmerman, A. J., Li, K. W., ... van Kesteren, R. E. (2014). Reducing hippocampal extracellular matrix reverses early memory deficits in a mouse model of Alzheimer's disease. Acta Neuropathologica Communications, 2, 76.

Villain, H., Benkahoul, A., Birmes, P., Ferry, B., \& Roullet, P. (2018). Influence of early stress on memory reconsolidation: Implications for posttraumatic stress disorder treatment. PLoS ONE, 13, e0191563.

Villain, H., Benkahoul, A., Drougard, A., Lafragette, M., Muzotte, E., Pech, S., ... Roullet, P. (2016). Effects of propranolol, a $\beta$-noradrenergic antagonist, on memory consolidation and reconsolidation in mice. Frontiers in Behavioral Neuroscience, 10, 1-14.

Warthen, D. M., Wiltgen, B. J., \& Provencio, I. (2011). Light enhances learned fear. Proceedings of the National Academy of Sciences of the United States of America, 108, 13788-13793.

Wood, N. E., Rosasco, M. L., Suris, A. M., Spring, J. D., Marin, M. F., Lasko, N. B., ... Pitman, R. 
K. (2015). Pharmacological blockade of memory reconsolidation in posttraumatic stress disorder: Three negative psychophysiological studies. Psychiatry Research, 225(1-2), 31-39.

Wotjak, C. T. (2019). Sound check, stage design and screen plot - how to increase the comparability of fear conditioning and fear extinction experiments. Psychopharmacology, 236, 33-48. 